\title{
The brightness of the red giant branch tip
} Theoretical framework, a set of reference models, and predicted observables

\author{
A. Serenelli ${ }^{1}$, A. Weiss ${ }^{2}$, S. Cassisi ${ }^{3}$, M. Salaris ${ }^{4}$, and A. Pietrinferni ${ }^{3}$ \\ 1 Institute of Space Sciences (IEEC-CSIC) Campus UAB, Carrer de Can Magrans, s/n, 08193, Barcelona, Spain \\ e-mail: aldos@ice.csic.es \\ 2 Max-Planck-Institut für Astrophysik, Karl-Schwarzschild-Str. 1, 86748 Garching, Germany \\ 3 INAF-Osservatorio Astronomico di Teramo, via M. Maggini, 64100 Teramo, Italy \\ 4 Astrophysics Research Institute, Liverpool John Moores University, IC2, Liverpool Science Park, 146 Brownlow Hill, Liverpool, \\ L3 5RF, UK
}

Received 18 April 2017 / Accepted 27 June 2017

\begin{abstract}
Context. The brightness of the tip of the red giant branch is a useful reference quantity for several fields of astrophysics. An accurate theoretical prediction is needed for such purposes.

Aims. We provide a solid theoretical prediction for the brightness of the tip of the red giant branch, valid for a reference set of standard physical assumptions, and mostly independent of numerical details.

Methods. We examine the dependence on physical assumptions and numerical details for a wide range of metallicities and masses and based on two different stellar evolution codes. We adjust differences between the codes to treat the physics as identically as possible. After we have succeeded in reproducing the tip brightness between the codes, we present a reference set of models based on the most up to date physical inputs, but neglecting microscopic diffusion, and convert theoretical luminosities to observed infrared colours suitable for observations of resolved populations of stars and include analytic fits to facilitate their use.

Results. We find that consistent use of updated nuclear reactions, including an appropriate treatment of the electron screening effects, and careful time-stepping on the upper red giant branch are the most important aspects to bring initially discrepant theoretical values into agreement. Small but visible differences remain unexplained for very low metallicities and mass values at and above $1.2 M_{\odot}$, corresponding to ages younger than $4 \mathrm{Gyr}$. The colour transformations introduce larger uncertainties than the differences between the two stellar evolution codes.

Conclusions. We demonstrate that careful stellar modelling allows an accurate prediction for the luminosity of the red giant branch tip. Differences to empirically determined brightnesses may result either from insufficient colour transformations or from deficits in the constitutional physics. We present the best-tested theoretical reference values to date.
\end{abstract}

Key words. stars: evolution - stars: interiors - stars: distances - methods: numerical - distance scale

\section{Introduction}

Stars of low mass, which are in the state of shell hydrogen burning and evolve along the first red giant branch (RGB), are of a comparably simple structure, which can be understood to a large extent by simple analytical relations. The method of shell source homology by Refsdal \& Weigert (1970) reproduces the strict connection between the mass of the degenerate, hydrogenexhausted helium core $\left(M_{\mathrm{c}}\right)$ and the stellar luminosity $(L)$, and with the temperature of the shell, which is a first-order approximation for the maximum temperature inside the core (see Kippenhahn et al. 2012, for a detailed derivation and further analytical relations). These relations are formally independent of the total stellar mass within a mass range of $\approx 0.5-2.0 M_{\odot}$, which is the classical definition of low-mass stars.

Owing to a rather narrowly defined helium-burning ignition temperature range close to $10^{8} \mathrm{~K}$, there is thus a very strong connection between core mass, core temperature, and luminosity. As the (off-centre) ignition of helium burning, known as the core helium flash, leads to a drastic change in the stellar interior, notably a complete lifting of degeneracy, the star quickly leaves the RGB and re-appears in the phase of quiescent central helium burning at lower luminosity and somewhat higher effective temperature on what is called either the red clump or, for metal-poor populations, the horizontal branch. This results in a sharp upper luminosity limit of the RGB, the tip of the red giant branch (TRGB).

A well-defined luminosity or absolute brightness for an easily identifiable class of objects, which, additionally, is only weakly dependent on composition and mass (or equivalently age), is an irresistible gift for astronomers. This is reinforced because, in particular, it also turned out that in suitable filters the luminosity of the TRGB is only weakly dependent on composition (Da Costa \& Armandroff 1990; Lee et al. 1993).

There are two major applications of this fact. The first and most obvious is to employ the TRGB brightness as a standard candle. For any given population of a sufficient number of stars, the TRGB can be easily detected in a resolved colour magnitude diagram (CMD) out to distances of several Mpc (e.g. Maíz-Apellániz et al. 2002) by using statistical filters to identify a break in the luminosity function (Sakai et al. 1996). It was shown repeatedly that the TRGB luminosity can compete in accuracy with other distance indicators such as Cepheids, surface brightness fluctuation, and the planetary nebulae luminosity 
function. It has become a standard rung in the extragalactic distance ladder, and has been used to determine distances of up to $10 \mathrm{Mpc}$ in over 40 cases (Jacobs et al. 2009).

The second method used to exploit the stability of the TRGB brightness uses the inverse approach: for a stellar system with well-known distance, the absolute brightness of the TRGB can be measured and compared to the theoretical predictions. This can identify deficits in the models, but the real benefit is to put bounds on additional, postulated physics, which would influence the details of helium ignition. The method is mostly applied to globular clusters and in connection with the properties of neutrinos and axions (Raffelt \& Weiss 1992, 1995; Catelan et al. 1996, and others). Here, additional cooling channels beyond the normal plasma neutrino emission require an increased core mass to achieve ignition temperatures and therefore, via the core mass-luminosity relation, a higher TRGB luminosity. The challenge for this method is the low number of stars on the upper RGB, which allows only for a statistical determination of the TRGB brightness. More recently, Viaux et al. (2013a,b) and Arceo-Díaz et al. (2015) have used the populous clusters M 5 and $\omega$ Cen for this purpose.

For both purposes, accurate and reliable theoretical predictions about the TRGB luminosity and consequently brightness in various photometric bands are needed. Stellar models are computed under a variety of options of physical effects, input data, and parameters. Examples are the inclusion or neglect of atomic diffusion, the various sources for opacities, and the mixinglength parameter for convection. Therefore, the dependence of the prediction on these choices is crucial as well. Raffelt \& Weiss (1992) and Viaux et al. (2013a) have studied the impact that theoretical uncertainties in stellar models have on photometric predictions, but only for one specific choice of stellar mass and composition in each work. We review this in Sect. 2 along with our earlier results and new calculations when appropriate. A further summary of the general dependency on mass and composition can be found in Salaris \& Cassisi (2006), and a presentation of the stability of the TRGB brightness in the $I$ band in Cassisi \& Salaris (2013).

In Sect. 3 we compare the results of two different stellar evolution codes, identify the reasons for the original discrepancies, and resolve them to a large degree. We present a representative set of models for well-defined, generic physics assumptions in Sect. 4 and achieve a satisfying agreement among them. This is followed by a discussion of the theoretical calculations in the context of using the TRGB as a distance indicator; we study the additional effect of the choice of bolometric corrections, and include comparisons of our results with empirical constraints in Sect. 5. Finally, in Sect. 6 we discuss the remaining uncertainties and the overall reliability of our reference predictions in the conclusions.

\section{Dependence of the TRGB brightness on input physics}

Owing to the existence of a well-established He core massluminosity relation for RGB models (see e.g. Cassisi \& Salaris 2013, and references therein), for any given initial chemical composition the TRGB bolometric luminosity $\left(L_{\mathrm{TRGB}}\right)$ is related to the mass size of the He core at the He-ignition $\left(M_{\mathrm{c}}^{\mathrm{He}}\right)$ :

$\frac{\partial \log \left(L_{\mathrm{TRGB}} / L_{\odot}\right)}{\partial \log \left(M_{\mathrm{c}}^{\mathrm{He}} / M_{\odot}\right)} \sim 6$.
This strong dependence implies that even a small change in the mass of the He core at the TRGB has a sizable effect on the luminosity of the models.

Theoretical $M_{\mathrm{c}}^{\mathrm{He}}$ values depend on input physics and on numerical assumptions in the model computations, as shown in the following. We start by discussing the effect on $M_{\mathrm{c}}^{\mathrm{He}}$ (and $L_{\mathrm{TRGB}}$ ) of varying, one at a time, the most relevant physics inputs by reviewing results available in the literature, covering mostly the cases of low-mass stars $\left(M<1 M_{\odot}\right)$ and low metallicities (typically $Z<0.006)$. We refer mostly to Catelan et al. (1996), Cassisi et al. (1998), Salaris \& Cassisi (2006), Cassisi \& Salaris (2013), Viaux et al. (2013b), Valle et al. (2013). If no reference is given, the results stem from unpublished tests made previously by the current authors.

In this work we have extended both mass and metallicity ranges, including models up to $1.4 M_{\odot}$ and $Z=0.04$ to include all possible cases for TRGBs as young as 4 Gyr. Results for this extended set of models are discussed in the following sections.

\subsection{Nuclear reaction rates}

Nuclear reaction rates are a key ingredient in the calculation of stellar models, and much effort has been devoted to improving the measurements of rates at energies as close as possible to the Gamow peak, i.e. the energies at which nuclear reactions occur in stars. Thanks to these studies, the reaction rates involved in the $p-p$ chain have been established with small uncertainties. The same is true for many reactions of the CNO-cycle, but for the ${ }^{14} \mathrm{~N}(\mathrm{p}, \gamma){ }^{15} \mathrm{O}$ rate, that could be still affected by some lingering uncertainty. This reaction is the slowest one in the whole cycle and therefore controls the $\mathrm{CNO}$-cycle efficiency. It is important in low-mass stars both near the end of core H-burning (Imbriani et al. 2004) and during the RGB evolution because $\mathrm{H}$-burning in the advancing shell is controlled by the CNO-cycle.

In the past, the rate for the ${ }^{14} \mathrm{~N}(\mathrm{p}, \gamma){ }^{15} \mathrm{O}$ reaction was affected by a significant uncertainty. The situation has improved thanks to the LUNA experiment (Formicola et al. 2004), which provided a more accurate determination of the cross section at relevant stellar energies. The updated rate is about a factor of two lower than older estimates, such as NACRE, that are still widely used in the literature (Angulo et al. 1999).

The impact of the LUNA rate on the He core mass and brightness at the RGB tip of low-mass stars has been investigated by Weiss et al. (2005) and Pietrinferni et al. (2010). Pietrinferni et al. (2010) found that the LUNA reaction rate leads to a larger value for $M_{\mathrm{c}}^{\mathrm{He}}$, of the order of $\approx 0.002-0.003 M_{\odot}$, with respect to the results obtained by using the NACRE rate. Despite the larger He core mass of the models based on the LUNA rate, their TRGB brightness is about $\Delta \log \left(L_{\mathrm{TRGB}} / L_{\odot}\right)=0.02$ lower because the lower $\mathrm{CNO}$-cycle efficiency in the H-burning shell of the LUNA models compensates for the luminosity increase expected from the larger core mass, as discussed by Weiss et al. (2005). The impact of this brightness change on the absolute I-Cousins magnitude of the TRGB is about 0.05 mag for a metallicity below $[\mathrm{Fe} / \mathrm{H}] \approx-1.0$, and even smaller at higher metallicities $^{1}$. In terms of simple analytical models, the effect can be understood as follows: for given luminosity $L$, the lower $C N O$ cycle efficiency requires a higher burning temperature in the shell. As this temperature sets the scale of the core temperature,

1 If the metal mixture is fixed in the models, and $[\alpha / \mathrm{Fe}]=0$, then $[\mathrm{Fe} / \mathrm{H}]=[\mathrm{M} / \mathrm{H}]=\log (\mathrm{Z} / \mathrm{X})-\log (\mathrm{Z} / \mathrm{X})_{\odot}$, where the solar value of the ratio $Z / \mathrm{X}$ is taken from the solar metal distribution adopted in the model calculations. 
the core will reach the He-ignition temperature at a lower luminosity (mass).

More recently this issue has again been investigated by Viaux et al. (2013b) who checked the impact on $M_{I}^{\mathrm{TRGB}}$ due to a variation of $\pm 15 \%$ of the ${ }^{14} \mathrm{~N}(\mathrm{p}, \gamma){ }^{15} \mathrm{O}$ reaction rate around the LUNA value. For their case study, an $M=0.82 M_{\odot}$ and $Z=0.00136$ model, they found this uncertainty to affect $M_{I}^{\mathrm{TRGB}}$ at the level of $\sim 0.009 \mathrm{mag}$, a weaker but consistent dependence, also in good agreement with the $M=0.9 M_{\odot}, Z=0.006$ model studied by Valle et al. (2013).

Since the TRGB marks the He ignition in the stellar core, another important nuclear process is the triple alpha reaction $3 \alpha \rightarrow{ }^{12} \mathrm{C}+\gamma$. The presence of resonances in the low energy range complicates an accurate estimate of this reaction rate. The most recent measurement has been obtained by Fynbo et al. (2005): they found a significant, temperature-dependent deviation from the older NACRE value, up to $-20 \%$ in the temperature range $70-100 \times 10^{6} \mathrm{~K}$ relevant to He-ignition in RGB cores. However, the difference decreases towards the upper temperature value, which is also the canonical He-ignition temperature (see Fig. 1 in Weiss et al. 2005). As a consequence, the impact of this new determination on the properties of TRGB models has turned out to be very small, as verified by Weiss et al. (2005) for low masses and low metallicities. The He core mass shows a very small increase, between 0.002 and $0.003 M_{\odot}$ (the exact value depending on the chemical composition and initial mass), and the TRGB luminosity increases by about $\Delta \log \left(L_{\mathrm{TRGB}} / L_{\odot}\right)=0.009$, i.e. by about $2 \%$, which translates to a $\Delta M_{I}^{\mathrm{TRGB}} \approx 0.02 \mathrm{mag}$. This is consistent with the result by Viaux et al. (2013b) who found, for their only test case, that a maximum uncertainty of $\sim 30 \%$ on the updated reaction rate would introduce a maximum $M_{I}^{\text {TRGB }}$ variation of about $0.02 \mathrm{mag}$. This increase in the TRGB luminosity is consistent with the expectation that a lower $3 \alpha$ rate requires a higher core temperature, i.e. a larger core mass, to initiate the core helium flash.

The bottom line of this analysis is that realistic residual uncertainties in these two reaction rates probably do not significantly affect the theoretical predictions of the TRGB absolute magnitude.

\subsection{Nuclear reaction screening}

Nuclear reactions inside the stars occur in a plasma where free electrons tend to distribute around the nuclei, shielding the Coulomb potential felt by the approaching particles. This implies that a correction - known as electron screening - has to be applied to properly evaluate the actual reaction rates in stellar models. Despite its relevance, the correct treatment of electron screening in a stellar plasma is still an unsettled issue (see e.g. Viaux et al. 2013b, for a discussion on this topic).

As a consequence, there is no proper estimate of the accuracy of the available electron screening predictions. An extreme test is to calculate models neglecting electron screening, to be compared with results obtained with the appropriate choice between the various levels of screening (strong, intermediate, and weak), determined by the available theoretical predictions (Dewitt et al. 1973; Graboske et al. 1973). The result of this experiment is that $M_{\mathrm{c}}^{\mathrm{He}}$ increases by $\approx 0.02 M_{\odot}$ when screening is neglected, corresponding to $\Delta \log \left(L_{\mathrm{TRGB}} / L_{\odot}\right) \approx 0.12$, hence about 0.30 mag in $M_{I}^{\mathrm{TRGB}}$.

\subsection{Equation of state}

The equation of state (EOS) is one of the most important ingredients in stellar model computations, and in the last decade significant improvements have been achieved in the description of the thermal conditions in the interiors and atmospheres of low-mass stars. Various EOSs currently available (see Viaux et al. 2013b, for a compilation) provide similar predictions; therefore, the use of different EOSs does not affect the TRGB. Indeed, Viaux et al. (2013b) have shown that when compared with models based on the most updated EOS (the FreeEOS ${ }^{2}$, Cassisi et al. 2003), the use of different EOS prescriptions introduces a maximum variation of +0.02 mag in $M_{I}^{\text {TRGB }}$.

Despite this result, it is in principle conceivable that for the highest density layers of the models, there could still be EOS uncertainties related to the treatment of the Coulomb and electron exchange effects. Accounting for different implementations of these processes in the EOS computation is quite difficult, but the FreeEOS code allows these non-ideal effects to be included or neglected. This has allowed us to estimate what is most probably an upper limit for the uncertainty associated with the EOS in the regime of RGB He cores ${ }^{3}$.

Calculations of low-mass star models neglecting the electron exchange effects show an increase in the He core mass at the TRGB by $\Delta M_{\mathrm{c}}^{\mathrm{He}}=9 \times 10^{-4} M_{\odot}$, and a decrease in the surface luminosity by $\Delta \log \left(L_{\mathrm{TRGB}} / L_{\odot}\right) \approx 0.003$. The decrease in the luminosity despite the (quite small) increase in the He core mass is due to the change in the inner temperature stratification which decreases the efficiency of the shell H-burning. Switching off the effects associated with Coulomb interactions in the EOS computations increases the He core mass by $\Delta M_{\mathrm{c}}^{\mathrm{He}}=4 \times 10^{-3} M_{\odot}$, while as in the previous case the luminosity at the He ignition decreases by $\Delta \log \left(L_{\mathrm{TRGB}} / L_{\odot}\right) \approx 0.003$. When both Coulomb and electron exchange effects are neglected in the EOS, the $\mathrm{He}$ core mass increases by about $0.0054 M_{\odot}$ and TRGB brightness is reduced by $\Delta \log \left(L_{\mathrm{TRGB}} / L_{\odot}\right) \approx 0.005$.

These tests show that lingering uncertainties in the EOS very likely have a negligible impact - if any at all - on predictions for the TRGB brightness. This is in line with the results by Viaux et al. (2013b).

\subsection{Radiative opacity}

Only the high-temperature $\left(T>10^{6} \mathrm{~K}\right)$ radiative Rosseland mean opacities can have some effect on the interior properties of RGB stars (Salaris et al. 1993), although opacities in the low-temperature regime affect the $T_{\text {eff }}$ of the models, hence the TRGB magnitudes through the indirect effect on the bolometric corrections (see Sect. 5).

In the electron degenerate He cores of evolved RGB stars the dominant energy transport mechanism is electron conduction, and as the models climb up the RGB, the contribution of the radiative opacity to the total opacity in the core becomes negligible in comparison with conductive opacity (see e.g. Salaris \& Cassisi 2006, and references therein). As a consequence, present uncertainties in high- $T$ radiative opacities should have a marginal impact on the TRGB properties of lowmass stars. Indeed, this expectation has been confirmed in the analysis made by Viaux et al. (2013b) who found that changing the radiative opacity by $\pm 10 \%$ causes a variation in $M_{I}^{\mathrm{TRGB}}$ of

\footnotetext{
2 http://freeeos.sourceforge.net/

3 A similar approach has been followed by Cassisi et al. (2003) to estimate the effect of EOS uncertainties on the theoretical predictions for the $R$-parameter.
} 
just $\approx \mp 3 \times 10^{-4}$. Currently, the uncertainty in the high- $T$ radiative opacities is believed to be of the order of $\sim 5 \%$, although there have been claims that this figure could reach a value of 20 $30 \%$ in some specific temperature ranges (see e.g. Catelan 2013, for a detailed discussion on this issue).

\subsection{Conductive opacity}

Electron conduction regulates the thermal state of the degenerate $\mathrm{He}$ core, and a reliable estimate of the conductive opacities $\left(\kappa_{\mathrm{cond}}\right)$ is fundamental in order to derive the correct value of $M_{\mathrm{c}}^{\mathrm{He}}$. As a rule of thumb, higher conductive opacities cause a less efficient cooling of the He-core and an earlier He-ignition, hence at a lower core mass and fainter TRGB brightness. The most recent $\kappa_{\text {cond }}$ update was provided by Cassisi et al. (2007), whose opacity set fully covers the thermal conditions characteristic of electron degenerate cores in low-mass metal-poor stars, accounts for arbitrary chemical mixtures, and takes into account the important contributions of electron-ion scattering and electron-electron scattering in the regime of partial electron degeneracy, which indeed is the relevant condition for the He cores of low-mass RGB stars. These new $\kappa_{\text {cond }}$ data result in values of $M_{\mathrm{c}}^{\mathrm{He}}$ that are lower than when using the previous calculations by Potekhin (1999); the difference amounts to $0.006 M_{\odot}$, regardless of the two tested metallicities. This difference in He-core mass at the RGB tip causes a TRGB that is fainter by $\Delta \log \left(L_{\mathrm{TRGB}} / L_{\odot} \approx 0.03\right.$ for the models based on the most recent $\kappa_{\text {cond }}$ values.

Again, it is difficult to estimate the remaining uncertainty in $\kappa_{\text {cond }}$ because of the complexity of the various physical processes whose combined effects determines the conductive transport efficiency. Viaux et al. (2013b) and Valle et al. (2013) assumed a $10 \%$ and $5 \%$ uncertainty, respectively, which resulted in variations of 0.016 and $0.007 \mathrm{mag}$ in $M_{I}^{\mathrm{TRGB}}$.

\subsection{Neutrino energy losses}

In the high temperature and density regimes characteristic of low-mass red giants, neutrino production through plasma-, photo-, pair-, and bremsstrahlung-processes, is quite efficient. These energy-loss channels play a crucial role in determining the value of the TRGB He core mass and brightness. In the dense and not extremely hot He core of RGB stars, plasma neutrino emission is the dominant process.

The most recent calculation of neutrino loss rates are still the ones provided by Haft et al. (1994) and Itoh et al. (1996a). Although Itoh et al. (1996b) claims that the accuracy of these rates is around 5\%, there is no clear proof that these predictions are unaffected by systematic errors. To estimate the impact that potential uncertainties in neutrino energy losses can have on lowmass stellar models, we compared the results based on the most recent evaluations, with calculations performed using the older Munakata et al. (1985) rates. To this purpose, we rely on the numerical experiments performed by Cassisi et al. (1998): these authors found that the use of the most updated neutrino energy losses - keeping all other input physics fixed - causes an increase in $M_{\mathrm{c}}^{\mathrm{He}}$ of about $0.006 M_{\odot}$, and a $\Delta \log \left(L_{\mathrm{TRGB}} / L_{\odot}\right) \approx 0.03$, corresponding to a variation in $M_{I}^{\mathrm{TRGB}}$ of $\approx-0.08 \mathrm{mag}$. This variation is about a factor of 6 larger than the effect estimated by Viaux et al. (2013b) by assuming a change of $\pm 5 \%$ in the neutrino emission rates by Haft et al. (1994).

We note that the present estimates of the plasma neutrino energy losses are based on the canonical assumption that neutrinos have no magnetic moment. If neutrinos had direct electromagnetic interactions due to the presence of a magnetic moment, the energy loss rates would be strongly enhanced, increasing the efficiency of neutrino cooling for larger magnetic moment. This would lead to an increase in the $\mathrm{He}$ core mass and luminosity at the TRGB. As mentioned already in the introduction, several investigations (Raffelt 1990; Castellani \& degl'Innocenti 1993; Viaux et al. 2013b,a) have been devoted to using the TRGB brightness in Galactic globular clusters to set upper limits for the neutrino magnetic moment (Viaux et al. 2013b). These investigations necessarily rely on the assumption that the known neutrino emission processes are treated with a sufficiently high accuracy.

\subsection{Diffusive processes}

Atomic diffusion (element transport due to temperature, pressure, and chemical abundance gradients) and radiative levitation (element transport due to the momentum imparted to ions by the outgoing photons) are potentially very important in lowmass stars as a consequence of their long main sequence lifetimes (see e.g. Cassisi \& Salaris 2013, for a detailed discussion on this topic and relevant references). As for the properties of the models at the TRGB, it is diffusion that can be important, as investigated by Cassisi et al. (1998) and by Michaud et al. (2010). If atomic diffusion is fully efficient during the main sequence phase, the TRGB $M_{\mathrm{c}}^{\mathrm{He}}$ increases by about $0.003-0.004 M_{\odot}$ for $Z \leq 0.001$; this variation slightly decreases when the metallicity increases. The increase in $M_{\mathrm{c}}^{\mathrm{He}}$ is a consequence of the small decrease $(\Delta Y \approx-0.01)$ in the He-abundance in the envelope during the RGB phase compared to models calculated without diffusion. This reduction changes the mean molecular weight, hence the efficiency of shell H-burning, delaying the ignition of the helium core (we recall that $L_{\mathrm{H}} \propto \mu^{7}$; Kippenhahn et al. 2012).

Owing to the larger He core mass, the TRGB brightness increases by $\Delta \log \left(L_{\mathrm{TRGB}} / L_{\odot}\right) \sim 0.003$ at $Z=0.0002$, and $\sim 0.01$ at $Z=0.002$; these variations translate to a change $\Delta M_{I}^{\mathrm{TRGB}}$ from -0.01 up to $-0.03 \mathrm{mag}$ (see also Viaux et al. $2013 \mathrm{~b}$, and references therein).

Finally, we note that the atomic diffusion velocities are generally thought to be accurate to a level of about 15-20\% (Thoul et al. 1994). However, it is difficult to account for these uncertainties in stellar model computations because this uncertainty might not be just a systematic error affecting all diffusion velocities of the various chemical elements (but see the analysis performed by Valle et al. 2013). We also note that the changes in the TRGB properties estimated above are likely to be upper limits because they are based on fully efficient atomic diffusion. Additional effects such as radiative levitation or extra-mixing are likely to diminish the net effect of atomic diffusion.

\section{Models and comparisons}

The dependence of $L_{\mathrm{TRGB}}$ on the input physics entering stellar model calculations is discussed in the previous section. In order to have a better assessment of the robustness of theoretical predictions for $L_{\mathrm{TRGB}}$, it is important to consider the consistency of results obtained with different evolutionary codes, which is discussed in this section, based on results obtained with the BaSTI code (Pietrinferni et al. 2004) and with GARSTEC (Weiss \& Schlattl 2008), in three different steps. Initially, we make a direct comparison of results obtained from calculations that were already available. Next, we define a concordance set of state-of-the-art input physics and compare stellar models newly 


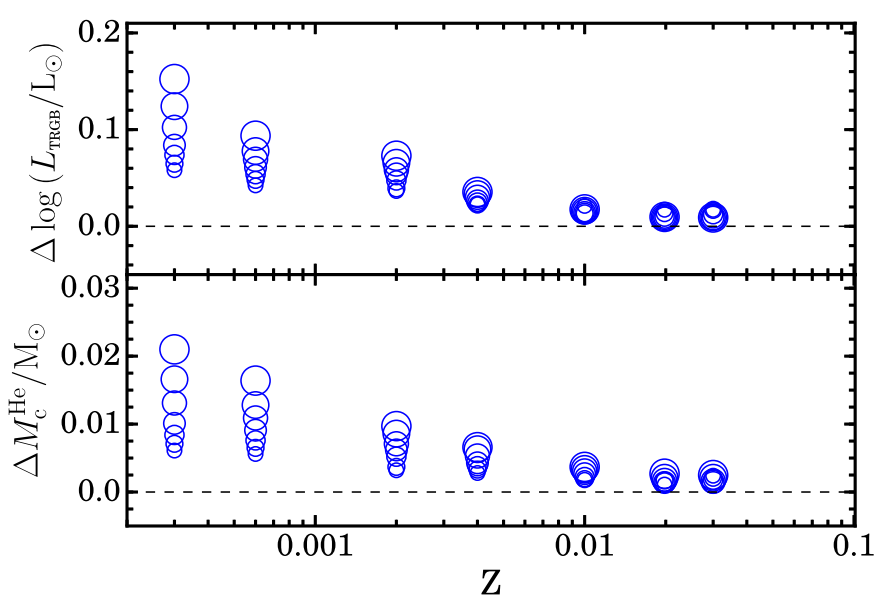

Fig. 1. Differences in the TRGB luminosity (top panel) and core mass at helium ignition (bottom panel) between two stellar codes as a function of metallicity. Symbol sizes denote the stellar mass, from $0.8 M_{\odot}$ to $1.4 M_{\odot}$ (smallest and largest symbols, respectively). Differences are in the sense BaSTI - GARSTEC.

computed based on this physics. Finally, we consider the influence of numerics that can differ among numerical codes and impact the predicted $L_{\mathrm{TRGB}}$.

\subsection{Initial comparison of two stellar code results}

The initial comparison is based on BaSTI results obtained using the physics described in Pietrinferni et al. (2004) and available at the BaSTI repository ${ }^{4}$, and on GARSTEC models computed using the physics described in Weiss \& Schlattl (2008). The most notable differences between the physics included in these models are the choice of the ${ }^{14} \mathrm{~N}(\mathrm{p}, \gamma){ }^{15} \mathrm{O}$ rate (NACRE for BaSTI and LUNA for GARSTEC), electron screening (Graboske et al. 1973, for BaSTI; and weak screening Salpeter 1954, for GARSTEC); and conductive opacity (Potekhin 1999, in BaSTI; and Cassisi et al. 2007, in GARSTEC).

In Fig. 1 the differences in $\Delta \log \left(L_{\mathrm{TRGB}} / L_{\odot}\right)$ and $M_{\mathrm{c}}^{\mathrm{He}}$ are given, in the sense that BaSTI - GARSTEC are shown as a function of metallicity and for a range of masses between 0.8 and $1.4 M_{\odot}$. At metallicities $Z>0.01$ there is good agreement across the whole mass range. This must be considered with some caution because the relevant physics is different in both sets of calculations. As we show later on, this agreement results from the compensation of differences between physics and numerics. At lower metallicity, differences increase substantially. Moreover, not only does the zero point agreement degrade to $0.05 \mathrm{dex}$ at $Z=0.0003$, but a large spread in stellar mass also appears. These results show that lower metallicities and larger masses imply higher sensitivity of the results on the input physics. For reference, in well-studied cases such as the globular cluster M 5 (Viaux et al. 2013b), the observational precision with which $L_{\mathrm{TRGB}}$ can be determined is about 0.04 dex.

\subsection{Two stellar codes, same physics}

Differences found in the previous section have two origins: different physical inputs and numerics in the stellar evolution codes. The next step is then to isolate each of these components. To this end, we have defined a set of concordance physics that

\footnotetext{
4 http://www.oa-teramo.inaf.it/BASTI/
}

represents at the same time the state-of-the-art physics and performed new ad hoc calculations with both BaSTI and GARSTEC.

In detail, we use low-temperature opacities from Ferguson et al. (2005), atomic radiative opacities from OPAL (Iglesias \& Rogers 1996), conductive opacities from Cassisi et al. (2007), the FreeEOS equation of state (Cassisi et al. 2003), neutrino energy losses from Haft et al. (1994) for plasma and from Munakata et al. (1985) updated to Itoh et al. (1996a) for other neutrino processes, and weak and intermediate nuclear screening following Dewitt et al. (1973) and Graboske et al. (1973). Nuclear reaction rates are not strictly the same. The GARSTEC models use the Solar Fusion II (SFII) rates (Adelberger et al. 2011) for all H-burning reactions and NACRE for the $3 \alpha$ reaction, the only relevant He-burning reaction in triggering He-core ignition. The basic set of H-burning reaction rates in BaSTI are from NACRE (Angulo et al. 1999) with the rate of ${ }^{14} \mathrm{~N}(\mathrm{p}, \gamma){ }^{15} \mathrm{O}$ updated to the recommended value from LUNA. The latter is different from the SFII value by only about $2 \%$, a minimal difference. Other rates linked to H-burning have a very minor influence on the luminosity at which helium is ignited. Therefore, despite the differences in the sources of the adopted nuclear reaction rates, in practice both codes use very similar values for the reactions relevant to this work. The set of model compositions we use follow those in Pietrinferni et al. (2004): $\Delta Y / \Delta Z \sim 1.4, Y=0.245$ at $Z=0$, and the metal mixture from Grevesse \& Noels (1993, hereafter GN93). Convection is modelled using the mixing length theory with solar calibrated parameters $\alpha_{\mathrm{MLT}}=1.83$ for GARSTEC and 2.02 for BASTI. The different calibration values are mainly due to the use of an Eddington atmosphere in GARSTEC and Krishna-Swamy in BASTI.

The impact of microscopic diffusion in the TRGB brightness is small. Results discussed in Sect. 2.7 show this even in the assumption that microscopic diffusion is fully operational, without considering possible processes such as radiative levitation, weak stellar wind during the main sequence phase, or extra-mixing below the convective envelope, all of which would diminish the effect of microscopic diffusion even further. While standard solar models routinely include microscopic diffusion (e.g. Vinyoles et al. 2017), it is also true that helioseismic constraints favour a smaller effective rate $(\sim 10-20 \%)$ than predicted by fully efficient diffusion models (Delahaye \& Pinsonneault 2006; Villante et al. 2014). The indication that diffusion is too efficient is even more compelling in metal-poor stars and has led to the introduction of ad hoc recipes for extra-mixing (Richard et al. 2002; VandenBerg et al. 2012). Therefore, after taking into account all these considerations, we chose not to include microscopic diffusion in our reference models that would have, nevertheless a very small impact on the results relevant to this work.

The models computed cover masses in the range $0.8-1.4 M_{\odot}$ and metal mass fractions $Z$ between $10^{-4}$ and 0.04 . This combination of parameters encompass all cases of interest in which the age of the TRGB is 4 Gyr or older. In fact, for the more massive and metal poor models the TRGB is reached in about 2 Gyr. Results for $\Delta \log \left(L_{\mathrm{TRGB}} / L_{\odot}\right)$ are shown in Fig. 2 as a function of metallicity, and a clear trend is present. However, there is an overall shift with respect to the initial comparison shown in Fig. 1. For $M \leq 1.2 M_{\odot}$, the agreement is better for the lowest metallicities, for which $L_{\mathrm{TRGB}}$ from the two codes agree to better than $0.02 \mathrm{dex}$, and is independent of stellar mass. This difference increases up to 0.07 dex at solar and higher metallicities. We note that the models of both codes change according to the expectations based on the dependencies discussed in Sect. 2, when changing the physical inputs to match the concordance physics. 


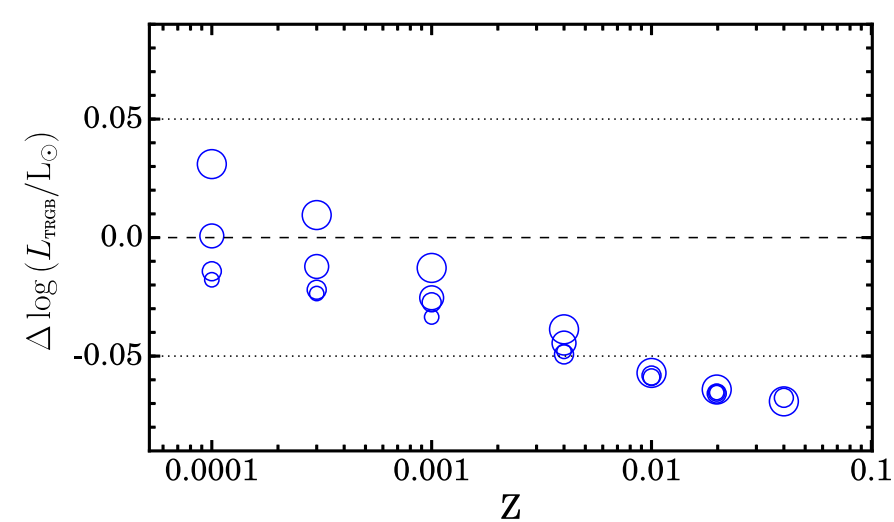

Fig. 2. Differences in TRGB luminosity as in top panel of Fig. 1, but for models computed using the concordance physics. GARSTEC models have been computed with $\Delta t_{\min }=3 \mathrm{kyr}$. Dotted lines indicate the estimated $1 \sigma$ theoretical uncertainty excluding electron screening (Sect. 2).

However, for masses above $M \leq 1.2 M_{\odot}$ there is an additional effect which leads to a spread in $\Delta L_{\mathrm{TRGB}}$, especially at $Z<0.001$. We come back to this below.

These differences, particularly at high metallicities, are larger than the combined uncertainty of the individual sources discussed in Sect. 2 as indicated in the plot, except for the extreme case of neglecting electron screening in nuclear reactions (Sect. 2.2). At high metallicities, they are also much larger than the level of observational uncertainties in the determination of the TRGB brightness.

\subsection{Numerical stability}

The final question we want to address is the impact that numerics involved in computation of stellar evolution models have on predictions related to the TRGB luminosity. Even for welldefined physics inputs, implementation in stellar evolution codes involves, more often than not, different numerical approaches. From interpolation of tables (equation of state, opacities) to the integration of the stellar structure equations (e.g. spatial and temporal resolution, convergence criteria), numerics have an impact on the models that stellar evolution codes provide.

We have checked the robustness of the $L_{\mathrm{TRGB}}$ predictions against changes in the integration time step used in stellar evolution calculations. This is controlled by limiting the fractional changes that several physical quantities can experience in one time step, e.g. temperature and pressure at a given mass coordinate, surface luminosity. Along the RGB, in particular, as the stellar luminosity increases, evolution speeds up and the integration time steps become progressively shorter, prompting the need to compute a larger number of models until the TRGB is reached. To avoid the calculation of too many stellar models, in GARSTEC it is possible to limit the smallest allowed time step, $\Delta t_{\min }$.

The results in Fig. 2 correspond to calculations where $\Delta t_{\text {min }}=3 \mathrm{kyr}$. Along the RGB, in typical GARSTEC calculations, the constantly decreasing time step reaches this $\Delta t_{\text {min }}$ value when $\log \left(L_{\mathrm{RGB}} / L_{\odot}\right) \approx 2$.7. For RGB calculations, BaSTI does not set a $\Delta t_{\min }$ value, and only changes in physical quantities determine the integration time step that reaches as low as $0.3 \mathrm{kyr}$ close to the TRGB. When a similar minimum time step is used in GARSTEC, the comparison between codes is much more satisfactory. This is shown in Fig. 3, where the top panel shows $\Delta \log \left(L_{\mathrm{TRGB}} / L_{\odot}\right)$ and the bottom panel differences in

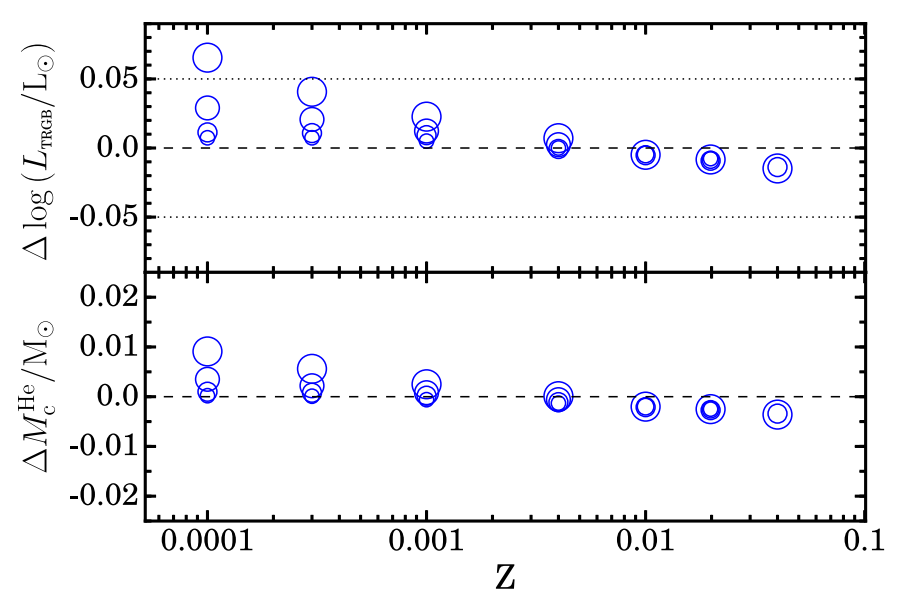

Fig. 3. Top panel: same as Fig. 2, but for $\Delta t_{\min }=0.3 \mathrm{kyr}$ in GARSTEC. Bottom panel: He-core mass difference.

$M_{\mathrm{c}}^{\mathrm{He}}$ between the two codes. For masses below 1.2 $M_{\odot}$ differences across all metallicities are now $\Delta \log \left(L_{\mathrm{TRGB}} / L_{\odot}\right)<0.02$, or $\Delta M_{I}^{\mathrm{TRGB}}<0.05$, well within the uncertainties imposed by the physics of stellar models. Core masses agree to better than $0.005 M_{\odot}$.

We carried out additional tests on the dependence of $L_{\mathrm{TRGB}}$ on the integration time steps used in the codes. The critical stage is the evolution along the higher RGB above $\log \left(L / L_{\odot}\right) \approx 2.6$, and small integration time steps have to be taken above this luminosity. In GARSTEC, taking $\Delta t_{\min }=1 \mathrm{kyr}$ is enough to yield robust predictions of $L_{\mathrm{TRGB}}$; reducing $\Delta t_{\text {min }}$ further, even down to $40 \mathrm{yr}$, leads to $\Delta \log \left(L_{\mathrm{TRGB}} / L_{\odot}\right) \approx 0.015$ or just 0.03 in $M_{I}^{\mathrm{TRGB}}$. With BaSTI results are similar; $L_{\mathrm{TRGB}}$ predictions converge nicely to within $\Delta \log \left(L_{\mathrm{TRGB}} / L_{\odot}\right) \approx 0.01$ for integration time steps that, at the end of the RGB, are smaller than a few hundred years.

Another important issue concerning numerical stability could be related to the choice of an appropriate number of mesh points in the model computations. This was tested by recent numerical experiments performed by one of the members of our group (S.C.): once the integration time step is fixed (in order to disentangle the effect of mesh resolution from that of time resolution) an increase of a factor of 2 in the number of spatial mesh points causes an increase in the He core mass at the RGB tip of only $\sim 0.0002 M_{\odot}$, and of the TRGB luminosity of $\Delta \log \left(L / L_{\odot}\right) \approx 0.002 \mathrm{dex}$; a further increase of a factor of 2 in the number of mesh points does not change the model predictions at the RGB tip. These effects being quite small, we do not investigate this issue further in the present work, and consider the standard spatial resolution to be sufficient.

Figure 3 shows that starting at $Z=0.004$, models with masses $\geq 1.2 M_{\odot}$ show increasing differences with decreasing metallicities. This is more noticeable for the $1.4 M_{\odot}$ models, the most massive ones shown in the figure, for which differences at the lowest metallcities exceed 0.05 dex. This trend is the same as in Fig. 2, and therefore does not depend on the time steps in this phase. In order to understand the origin of this discrepancy we carried out some additional tests. We tested whether different sizes of the convective cores during the main sequence could affect the RGB-tip luminosity for these models. However, convective cores for these masses and metallicities are rather small and bear no impact on the RGB evolution. We also considered the different implementation of radiative and conductive opacities in GARSTEC and BASTI, particularly in the high density regions and different interpolation schemes for radiative opacity 
(e.g. linear in $Z$, linear in $\log Z$ ). Some differences in the final opacities are present in both codes and can be traced back to slightly different implementations of the conductive opacities. These differences are quite small, with GARSTEC being systematically lower than BASTI, but the temperature-density profile in the $1.4 M_{\odot}$ model is very similar to that in the $0.8 M_{\odot}$ model, for which the two codes agree very well. In fact, as can be inferred from Sect. 2.5, a 10\% change in conductive opacities leads to a $\Delta \log \left(L_{\mathrm{TRGB}} / L_{\odot}\right) \sim 0.006 \mathrm{dex}$, much lower than the difference we find for the $1.4 M_{\odot}$ model with $Z=0.0001$. Therefore, we conclude that the systematic opacity difference cannot be the reason for the different level of agreement between models of different masses at low metallicities. We were not able to find the reason for this discrepancy and it certainly requires further consideration. Fortunately, in the context of this work, it corresponds to low-metallicity stars that reach the TRGB in less than 3 Gyr, i.e. in a regime that makes observational interest rather limited.

\section{Standard set of models}

The concordance physics used in the previous section was chosen not only because it is available both in GARSTEC and BaSTI, but also because it represents state-of-the-art inputs appropriate for low-mass stellar models, keeping in mind the uncertainties mentioned in Sect. 3.2 regarding microscopic diffusion. We therefore adopt it as the input physics in the standard set of models we use in the remainder of this paper and that have been computed with GARSTEC. In terms of numerics, models have been computed with a $\Delta t_{\min }=1 \mathrm{kyr}$ minimum allowed time step along the RGB, the least restrictive time step that is able to reduce numerical uncertainties well below those from physical ingredients in the models. The mass and metallicity ranges covered by the standard set of models are those used for the intercode comparison, i.e. $M$ between 0.8 and $1.4 M_{\odot}$ and $Z$ between $10^{-4}$ and 0.04 , but with a higher density of models, aimed at including all possible cases the TRGBs that are 4 Gyr or older.

The composition adopted in our reference models is based on the GN93 solar composition and does not include $\alpha$ enhancement. At fixed $Z$, differences in $L_{\mathrm{TRGB}}$ and $T_{\mathrm{eff}}^{\mathrm{TRGB}}$ between models with $[\alpha / \mathrm{Fe}]=0$ and 0.4 is less than $1 \%$ and $2 \%$, respectively, for masses up to $1.5 M_{\odot}$ and up to solar metallicities. Moreover, as shown in Cassisi et al. (2004), colour transformations in the infrared filters, $I$, and $(V-I)$ are the same for solar-scaled and $\alpha$-enhanced compositions at either the same $[\mathrm{Fe} / \mathrm{H}]$ or $[\mathrm{M} / \mathrm{H}]$, with differences being at most $0.01 \mathrm{mag}$ at fixed colour in the $V I$ and $J K$ colour magnitude diagrams (see next sections).

The choice of $Y$ in our models assumes a primordial mass fraction of 0.245 . Current estimates range from approximately this value up to 0.256 (see Cyburt et al. 2016, and references therein). A variation of $\Delta Y=0.01$ has a very small impact on the TRGB properties at all metallicities and masses of interest in our work, with changes smaller than $1 \%$ in $L_{\mathrm{TRGB}}$ and $0.5 \%$ in $T_{\mathrm{eff}}^{\mathrm{TRB}}$. Therefore, such small variations in $Y$ can be safely neglected as relevant sources of uncertainties. A similar argument follows for our choice $\Delta Y / \Delta Z \sim 1.4$ because varying $\Delta Y / \Delta Z$ in the range from 1 to 2 leads to changes in $Y$ of about 0.01 at solar metallicities and, logically, smaller at lower metallcities. Figure 4 shows several of the evolutionary tracks corresponding to our standard set of models, for different metallicities and masses, from the zero age main sequence (ZAMS) up to the TRGB.

With respect to previous works exploring uncertainties in the determination of the TRGB luminosity and photometric

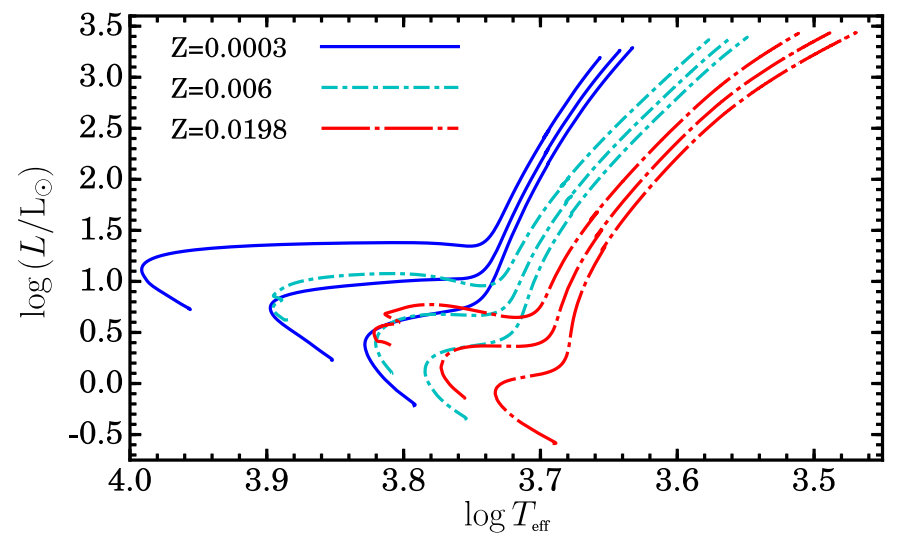

Fig. 4. Evolutionary tracks extending from the ZAMS up to the TRGB for some of our standard set of models. For each metallicity, masses are $0.8,1$, and $1.3 M_{\odot}$

properties discussed in Sect. 2, we have examined the full mass and metallicity ranges to assess uncertainties in CMDs for different choices of photometric bands, complementing the set of standard models by additional sets of models with varied physics. The photometric properties of these additional models are presented in the next section as well (see Fig. 7).

\section{TRGB as distance indicator}

Indications of the possibility of using the TRGB as a distance indicator can be traced back to Baade (1944) and Sandage (1971), but it started to be used for distance determinations only in the early eighties (see a summary in Lee et al. 1993), and the method was formalized by Lee et al. (1993), who proposed the use of an edge-detection algorithm to identify objectively the TRGB in the CMD of galaxies. A thorough analysis of the technique and estimates of statistical uncertainties due to low number statistics followed in Madore \& Freedman (1995). Alternative parametric and non-parametric methods have also been applied to determine the TRGB magnitude in a number of stellar systems (see e.g. Salaris \& Cassisi 1998; Cioni et al. 2000; Makarov et al. 2006; Conn et al. 2011).

Irrespective of the technique employed to determine the observed TRGB magnitude, the use of the TRGB as distance indicator has been traditionally based on I-band photometry, on the assumption that the observed RGB of a given stellar system has an age comparable to the age of Galactic globular clusters, or in any case larger than 4-5 Gyr (see e.g. Da Costa \& Armandroff 1990; Lee et al. 1993; Salaris et al. 2002). The reason is that in this age range $M_{\mathrm{bol}}^{\mathrm{TRGB}}$ is roughly constant at a given $[\mathrm{M} / \mathrm{H}]$ and for $[\mathrm{M} / \mathrm{H}]$ ranging between $\sim-2.0$ and $\sim-0.7, M_{\mathrm{bol}}^{\mathrm{TRGB}}$ is proportional to $\sim-0.18[\mathrm{M} / \mathrm{H}]$, whilst the bolometric correction to the $I$ band $B C_{I}$ is proportional to $\sim-0.24(V-I)$ (Da Costa \& Armandroff 1990). Given that the $(V-I)$ colour of the TRGB varies approximately as $0.57[\mathrm{M} / \mathrm{H}], B C_{I}$ is then proportional to $\sim-0.14[\mathrm{M} / \mathrm{H}]$, hence $M_{I}^{\mathrm{TRGB}}=M_{\mathrm{bol}}^{\mathrm{TRGB}}-B C_{I}$ is almost independent of $[\mathrm{M} / \mathrm{H}]$. Values of $M_{I}^{\mathrm{TRGB}} \sim-4.0$ are generally employed without or with a mild dependence on $[\mathrm{M} / \mathrm{H}]$ (see e.g. the theoretical calibration by Salaris \& Cassisi 1997).

As discussed in Barker et al. (2004), Salaris \& Girardi (2005), and Cassisi \& Salaris (2013), applying this type of TRGB calibrations to galaxies with a star formation that reaches ages below 4-5 Gyr and/or metallicities above $[\mathrm{M} / \mathrm{H}] \sim-0.7$ can cause sizable systematic errors on their derived distance, as recently confirmed empirically by Górski et al. (2016). To 


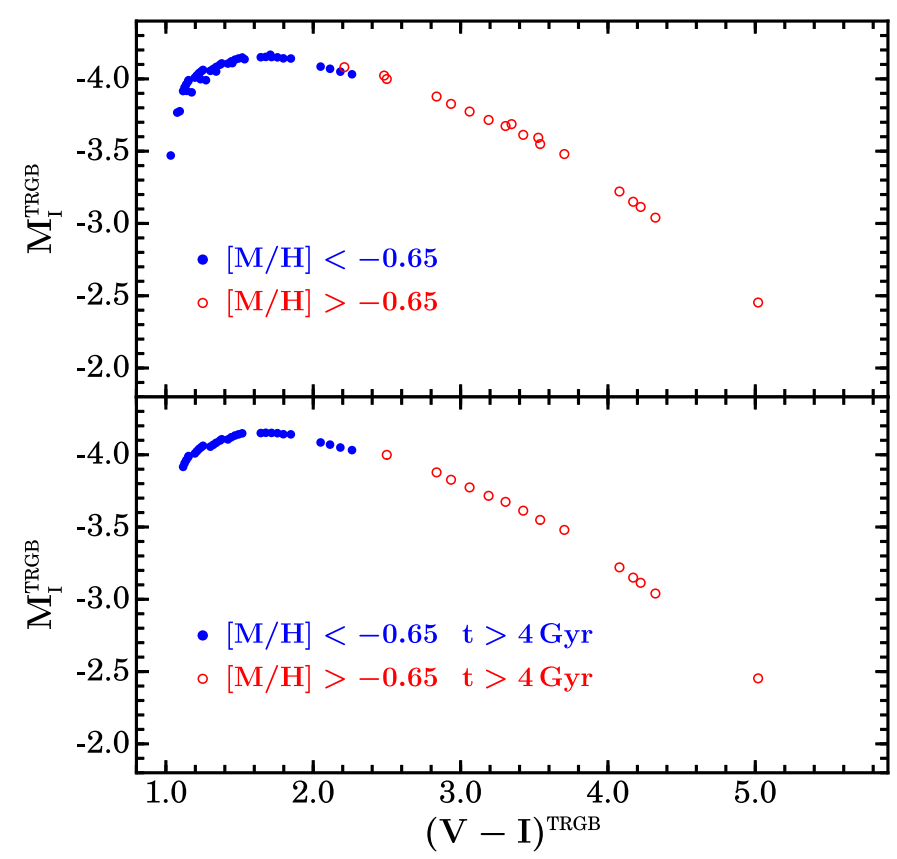

Fig. 5. Theoretical $M_{I}^{\mathrm{TRGB}}-(V-I)^{\mathrm{TRGB}}$ relation covering the full age and $[\mathrm{M} / \mathrm{H}]$ range of our reference calculations (upper panel), and considering only ages above $4 \mathrm{Gyr}$ (lower panel). Two different $[\mathrm{M} / \mathrm{H}]$ ranges are denoted with different symbols, as labelled.

circumvent this problem it is appropriate to use a $M_{I}^{\mathrm{TRGB}}-(V-$ $I)^{\mathrm{TRGB}}$ relation whereby the TRGB colour essentially accounts for the star formation history of the observed population. A relationship of this type appears to be smooth and tight, both empirically (Rizzi et al. 2007) and theoretically, as shown below.

Figure 5 displays the theoretical $M_{I}^{\mathrm{TRGB}}-(V-I)^{\mathrm{TRGB}}$ calibration in Johnson-Cousins filters (here and in the following plots we consider ages lower than $\sim 15 \mathrm{Gyr}$, discarding points corresponding to unrealistically old ages) derived from our "best choice" calculations (Sect. 4), after applying the empirical bolometric corrections by Worthey \& Lee (2011). The upper panel displays with different symbols the TRGB magnitude and colour for $[\mathrm{M} / \mathrm{H}]$ higher (up to $[\mathrm{M} / \mathrm{H}] \sim 0.4$ ) and lower (down to a lower limit $[\mathrm{M} / \mathrm{H}] \sim-2.3)$ than -0.65 . In general, decreasing age at constant $[\mathrm{M} / \mathrm{H}]$ shifts $M_{I}^{\mathrm{TRGB}}$ to bluer colours, while increasing $[\mathrm{M} / \mathrm{H}]$ at fixed age has the opposite effect. The overall shape of the $M_{I}^{\mathrm{TRGB}}-(V-I)^{\mathrm{TRGB}}$ relation displays a maximum brightness around $(V-I)^{\mathrm{TRGB}} \sim 1.7 \mathrm{mag}$, corresponding to $[\mathrm{M} / \mathrm{H}] \sim-1$ and ages between $\sim 8 \mathrm{Gyr}$ and $\sim 12 \mathrm{Gyr}$. For $(V-I)^{\mathrm{TRGB}}$ below $\sim 1.1 \mathrm{mag}$ (corresponding to $[\mathrm{M} / \mathrm{H}]=-2.3$ and ages below $4 \mathrm{Gyr}$ ), $M_{I}^{\text {TRGB }}$ increases at almost constant colour, whilst above $(V-I)^{\mathrm{TRGB}} \sim 1.7$ the TRGB magnitude increases with colour much more slowly.

The lower panel of Fig. 5 displays with different symbols TRGB magnitudes and colours for ages above $4 \mathrm{Gyr}$ with $[\mathrm{M} / \mathrm{H}]>-0.65$ and $[\mathrm{M} / \mathrm{H}]<-0.65$ (this latter being the "classical" TRGB calibration for distance determinations).

The classical calibration with our reference models provides a median $M_{I}^{\mathrm{TRGB}}=-4.07$ with a total range of $\pm 0.08 \mathrm{mag}$ around this value. The behaviour of $M_{I}^{\mathrm{TRGB}}$ vs. $(V-I)^{\mathrm{TRGB}}$ in this age and metallicity range is approximately quadratic, but assuming a constant average value of $M_{I}^{\mathrm{TRGB}}$ is still a decent approximation, as shown by the relatively small range of values spanned by $M_{I}^{\mathrm{TRGB}}$.

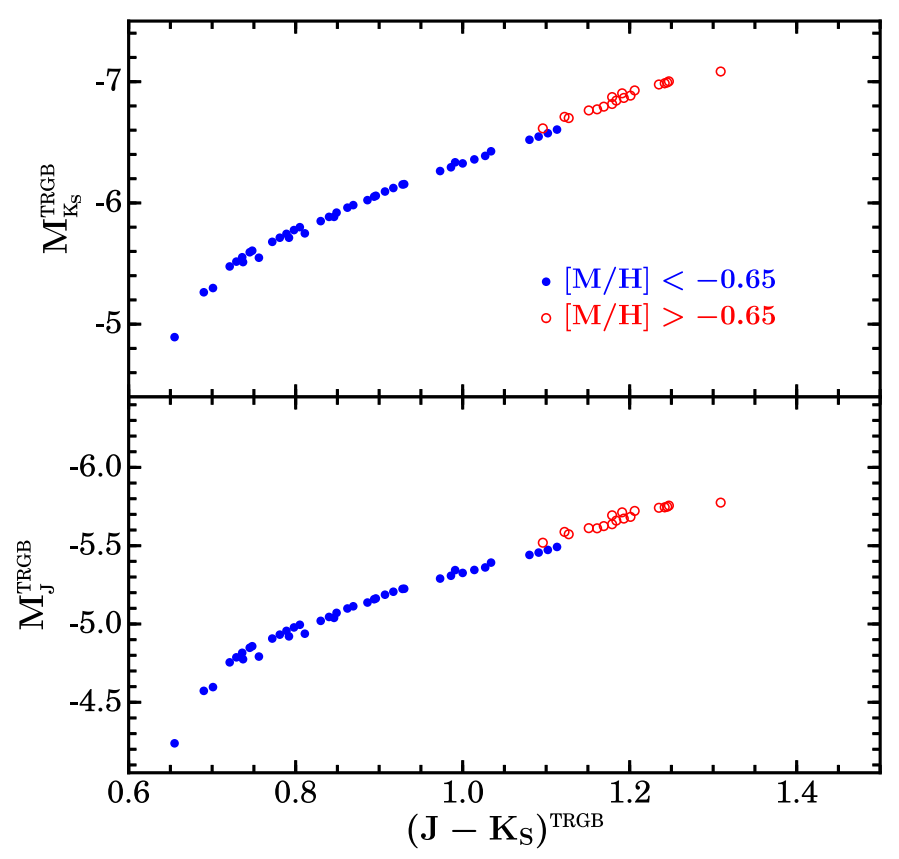

Fig. 6. As the upper panel of Fig. 5 , but for the $M_{K_{\mathrm{S}}}^{\mathrm{TRGB}}-\left(J-K_{\mathrm{s}}\right)^{\mathrm{TRGB}}$ (upper panel) and $M_{J}^{\mathrm{TRGB}}-\left(J-K_{\mathrm{s}}\right)^{\mathrm{TRGB}}$ relations (lower panel) in the 2MASS photometric system.

It is evident that the $M_{I}^{\mathrm{TRGB}}-(V-I)^{\mathrm{TRGB}}$ relationship is very smooth, irrespective of any $[\mathrm{M} / \mathrm{H}]$ and age selection, and for $(V-I)^{\mathrm{TRGB}}$ larger than $\sim 1.1 \mathrm{mag}$ it can be used as the distance indicator of any stellar population that displays a well-populated RGB.

The same holds true in infrared filters. Figure 6 displays $M_{K_{\mathrm{S}}}^{\mathrm{TRGB}}-\left(J-K_{\mathrm{s}}\right)^{\mathrm{TRGB}}$ and $M_{J}^{\mathrm{TRGB}}-\left(J-K_{\mathrm{s}}\right)^{\mathrm{TRGB}}$ relations in the 2MASS photometric system, derived from our reference calculations and the bolometric corrections by Worthey \& Lee (2011), after applying the Carpenter (2001) transformations from the Bessell-Brett system to the 2MASS system. The magnitudecolour relationships are again very smooth, with the added bonus of a lower sensitivity to reddening and a much more linear behaviour compared to the VI bands. We also note how the range of the TRGB absolute magnitude in $J$ is reduced compared to the case of the $K$ band. Wu et al. (2014) have also derived empirical tight and smooth relationships in the corresponding infrared filters of the WFC3-HST system $(F 110 W$ and $F 160 W)$.

In the following we consider $V I$ and $J K$ absolute magnitudecolour relationships as the most reliable way to determine TRGB distances, and discuss the uncertainties in their theoretical calibration stemming from stellar models and from bolometric correction uncertainties.

\subsection{Theoretical uncertainties on TRGB distances: stellar models}

To discuss the effect of varying stellar input physics on theoretical TRGB distance calibrations, we calculated additional sets of models by varying one "ingredient" at a time compared to the reference GARSTEC set based on the physics inputs listed in 3.2, with a solar calibrated mixing length $\alpha_{\mathrm{MLT}}$ and $\Delta t_{\min }=1 \mathrm{kyr}$.

Specifically, the additional sets include these changes:

1. variation of the $3 \alpha$ reaction rate by $\pm 10 \%$; 


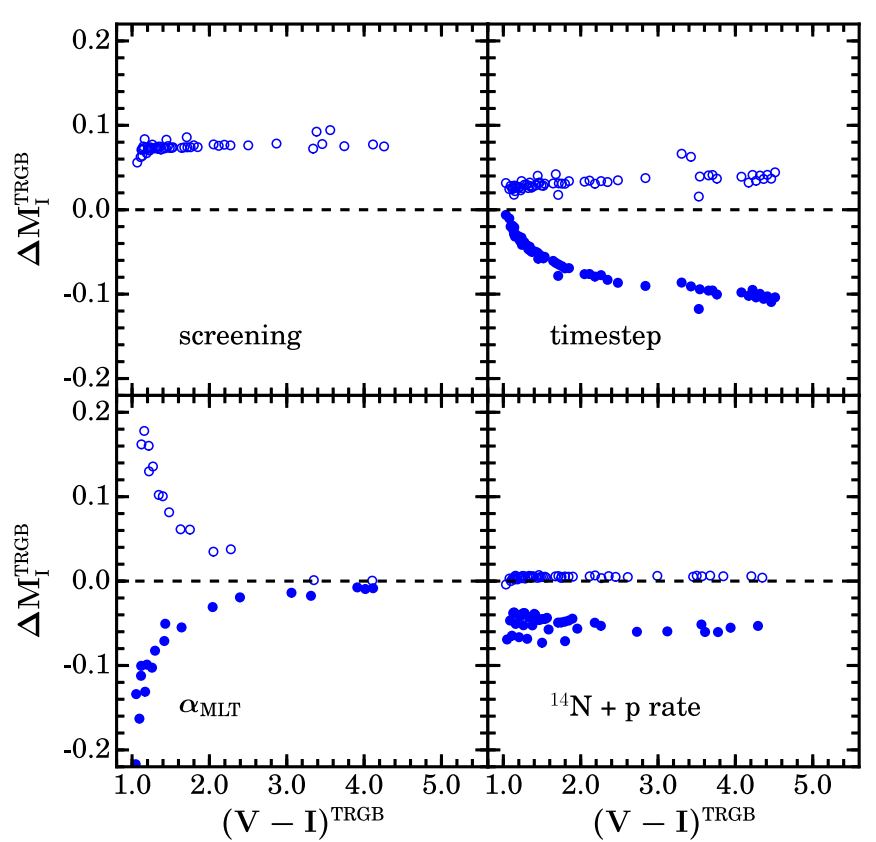

Fig. 7. Difference of $M_{I}^{\mathrm{TRGB}}$ at fixed $(V-I)^{\mathrm{TRGB}}$ colour when varying - one at a time - the labelled physics inputs and computational time steps (see text for details). Filled circles in the various panels display the cases with $\Delta t_{\min }=3 \mathrm{kyr}$ time steps, $\Delta \alpha_{\mathrm{MLT}}=+0.2$, and NACRE reaction rates for all nuclear reactions (including the ${ }^{14} \mathrm{~N}(\mathrm{p}, \gamma){ }^{15} \mathrm{O}$ reaction). Open circles display the cases of weak screening, $\Delta t_{\min }=0.3 \mathrm{kyr}$, $\Delta \alpha_{\mathrm{MLT}}=-0.2$, and NACRE rates plus the LUNA rate for the ${ }^{14} \mathrm{~N}+\mathrm{p}$ reaction.

2. use of NACRE reaction rates instead of those by SFII, but the LUNA rate for the ${ }^{14} \mathrm{~N}(\mathrm{p}, \gamma){ }^{15}$ reaction;

3. use of NACRE rates for all reactions;

4. weak screening instead of the appropriate value;

5. variation of the mixing length $\alpha_{\mathrm{MLT}}$ by \pm 0.2 .

Figure 7 compares the corresponding variations of $M_{I}^{\mathrm{TRGB}}$ (the variations of the $K_{\mathrm{s}}$ and $J$ TRGB magnitudes at fixed $(J-K)$ follow the same qualitative and quantitative pattern). The displayed differences are in the sense TRGB with varied input - reference GARSTEC TRGB, and have been determined by interpolating the results for the reference model set to the colours of each of the calculations with varied inputs. We do not show the results for the first case because the variations of the TRGB brightness are essentially zero. We add instead the case of models calculated with $\Delta t_{\min }=3 \mathrm{kyr}$ (standard GARSTEC calculations) and $0.3 \mathrm{kyr}$, presented in Sect. 3.3.

The choice of the LUNA rate for the ${ }^{14} \mathrm{~N}(\mathrm{p}, \gamma){ }^{15}$ reaction and NACRE rates for all other reactions has a negligible effect, at the level of $\sim 0.01 \mathrm{mag}$ on average. In the case of NACRE rates also for the critical ${ }^{14} \mathrm{~N}(\mathrm{p}, \gamma)^{15}$ reaction the impact is, on average, at the level of $0.05 \mathrm{mag}$ for both $V I$ and $J K$ calibrations, the TRGB being brighter if the NACRE rate is employed.

The choice of the time step and treatment of electron screening have also an appreciable effect. The use of weak screening instead of the appropriate choice between strong, intermediate, and weak cases, makes the TRGB fainter (because of smaller $\mathrm{He}$-core masses at the He-flash) by typically around $0.08 \mathrm{mag}^{5}$. As for the time step, the standard $\Delta t_{\min }=3 \mathrm{kyr}$ provide TRGB

Weak, intermediate, and strong refer to the strength of the Coulomb interaction between neighbouring nuclei compared to the thermal energy, but actually weak screening enhances reaction rates more strongly than intermediate screening in RGB cores. magnitudes systematically brighter (at fixed colour) compared to the reference results with $\Delta t_{\min }=1 \mathrm{kyr}$. There is a clear strong trend of these differences with colour, in the sense of larger differences (up to $\sim 0.10 \mathrm{mag}$ ) for redder colours (higher metallicities). The difference with results for models calculated with the shortest time steps is very small, of the order of just 0.03 mag on average (see also the discussion in Sect. 3.3).

Finally, the effect of mixing length variations is never negligible. Given that the bolometric luminosity of the TRGB is unaffected by the choice of $\alpha_{\text {MLT }}$, the differences seen in Fig. 7 are due to the change in the TRGB effective temperature with changing $\alpha_{\mathrm{MLT}}$, and the resulting change in colours and bolometric corrections. In practice, variations of $\alpha_{\text {MLT }}$ can be understood as a proxy for uncertainties in the $T_{\text {eff }}$ scale of RGB models. As discussed in Cassisi (2010), these uncertainties are of the order of 200-300 K, corresponding to $\Delta \alpha_{\mathrm{MLT}} \sim 0.20-0.30$, depending on the metallicity of models. Therefore, by considering a range $\Delta \alpha_{\mathrm{MLT}}= \pm 0.2$, we set a rather conservative estimate of the $T_{\text {eff }}$ uncertainty on the absolute magnitude of the TRGB. The decrease in $\alpha_{\text {MLT }}$ makes the TRGB fainter in the I and J bands (the opposite is true for an increase in $\alpha_{\mathrm{MLT}}$ ), the magnitude change increasing steeply with decreasing colour, up to $\sim 0.2 \mathrm{mag}$. As stated above, this is probably an overestimate of the true uncertainty, which is more likely to be \pm 0.1 or smaller (lower left panel in Fig. 7).

These systematic effects are roughly additive, so that the combination " $\Delta t_{\min }=0.3 \mathrm{kyr}$ " + "weak screening" + "Adelberger et al. (2011) ${ }^{14} \mathrm{~N}+\mathrm{p}$ rate" + "increased $\alpha_{\text {MLT }}$ " would provide TRGB magnitudes on average $\sim 0.25-0.30 \mathrm{mag}$ fainter than the combination " $\Delta t_{\min }=3 \mathrm{kyr}$ " + "full screening" + "NACRE ${ }^{14} \mathrm{~N}+\mathrm{p}$ rate" + "decreased $\alpha_{\mathrm{MLT}}$ " at the extreme ends of the magnitude-colour calibration.

Our best choice set of TRGB models is the one defined in Sect. 4, and provides TRGB magnitudes intermediate between the brighter and the fainter combinations described above.

An additional potential source of systematics for theoretical TRGB magnitude-colour calibrations is the choice of the bolometric corrections (BCs) applied to the TRGB bolometric magnitudes and effective temperatures predicted by stellar model calculations.

\subsection{Theoretical uncertainties on TRGB distances: bolometric corrections}

In the case of the model calculations it is possible, at least in principle, to identify the best combination of input physics/numerics, whereas it is extremely difficult to select a priori the best set of BCs. In the following we will consider the empirical BCs by Worthey \& Lee (2011), which we have already used in the previous plots, plus the semiempirical BCs by Westera et al. (2002) and the theoretical results from the MARCS (Gustafsson et al. 2008) and PHOENIX (Dotter et al. 2008) model atmospheres ${ }^{6}$. The Westera et al. (2002) BCs do not include metallicities [M/H] below -2.0, whilst the MARCS results do not include surface gravities $\log (g)<0.0$; therefore, they do not cover the oldest solar metallicity TRGBs and the supersolar ones.

Figures 8 and 9 compare the $V I$ and $J K$ absolute magnitudecolour calibrations obtained with our best choice theoretical models and these sets of BCs. The differences are not negligible and become substantial in certain colour ranges. In the case

\footnotetext{
6 For the MARCS BCs we employed the routine provided by Casagrande \& VandenBerg (2014), whereas the PHOENIX BCs were provided by A. Dotter (priv. comm.).
} 
of the $M_{I}^{\mathrm{TRGB}}-(V-I)^{\mathrm{TRGB}}$ calibrations, $M_{I}^{\mathrm{TRGB}}$ differences at fixed $(V-I)^{\mathrm{TRGB}}$ increase with increasing colours. When $(V-I)^{\mathrm{TRGB}}$ is between $\sim 2.5$ and $\sim 4.0$ ([M/H] approximately between -0.6 and solar) the differences reach $\sim 0.3$ mag, whilst at bluer colours they are typically of the order of $0.1-0.15 \mathrm{mag}$. Above $(V-I)^{\mathrm{TRGB}} \sim 4.0$ the slopes of the magnitude-colour relationships are radically different. The Westera et al. (2002) BC produce sizable discontinuities in the magnitude-colour relationship due to the stronger dependence of the $\mathrm{BC}$ to the $I$-band on metallicity, compared to the other sets of BCs.

A word of caution is necessary regarding the use of VI. The point where He-core ignition occurs always corresponds to the point with the largest bolometric luminosity, but this does not necessarily coincide with the brightest point in the $I$ band, i.e. the TRGB, because of the dependence of the BC on effective temperature. In fact, the He-core ignition point and the TRGB are different with the Westera transformation for $(V-I)>3.0$ (the point of He-core ignition is fainter than the TRGB), while for the Worthey \& Lee transformation the He-flash always corresponds to the TRGB. For the MARCS models, the brightest TRGB in the $I$ band is reached around $T_{\text {eff }} \sim 3400 \mathrm{~K}$, with the TRGB becoming dimmer in the $I$ band at lower $T_{\text {eff }}$. Therefore, depending on the set of BCs used, the He-flash can be fainter than the TRGB and hence, from a theoretical point of view, the TRGB is much less well-defined when using $V I$ photometry. Observationally, the $M_{I}-(V-I)$ diagram of 47 Tuc in Bellazzini et al. (2004) shows an almost constant $M_{I}$ at the reddest $V-I$ colours and indicates that this caveat is relevant for old populations more metal rich than $[\mathrm{Fe} / \mathrm{H}] \approx-0.5$.

In the infrared filters the various sets of BCs produce different overall slopes of the magnitude-colour relationships. Differences of $M_{J}^{\mathrm{TRGB}}$ or $M_{K}^{\mathrm{TRGB}}$ at fixed $(J-K)^{\mathrm{TRGB}}$ increase towards larger colours (higher metallicities), reaching $\sim 0.30$ mag in $J$ and $K$ at $\left(J-K_{\mathrm{s}}\right)^{\mathrm{TRGB}}>1.0-1.1([\mathrm{M} / \mathrm{H}]$ above $\sim-0.65)$. The closest agreement is in the colour range $0.8<\left(J-K_{\mathrm{s}}\right)<1.0$, where differences are at most of the order of $\sim 0.1 \mathrm{mag}$. Below $\left(J-K_{\mathrm{S}}\right)^{\mathrm{TRGB}} \sim 0.8$ (corresponding to $[\mathrm{M} / \mathrm{H}] \sim-1.5$ to $-1.8, \mathrm{de}-$ pending on age) the effect of the choice of BCs increases again, up to $\sim 0.2 \mathrm{mag}$. In $J K$ photometry, the He-flash is always the brightest point, i.e. it always coincides with the TRGB at all $(J-K)$ colours for these sets of BCs.

Existing empirical constraints on the TRGB brightness do not help discriminate amongst the various sets of BCs in the $V I$ diagram, and actually highlight inconsistencies, as shown by Fig. 10, which displays the $M_{I}^{\mathrm{TRGB}}-(V-I)^{\mathrm{TRGB}}$ calibrations of Fig. 8 together with the empirical estimate for the Galactic globular cluster $\omega$ Cen (Bellazzini et al. 2001, 2004) based on the cluster eclipsing binary distance. The error bar on $M_{I}^{\mathrm{TRGB}}$ is still substantial and cannot put strong constraints on the theoretical calibration. We do not include an analogous estimate for the more metal-rich Galactic globular 47 Tuc - because of large error bars and the need to apply theoretical corrections to infer the real TRGB location from observations - due to the paucity of bright RGB stars that bias the TRGB detection (see Bellazzini et al. 2004, for details).

In the same figure we also display the empirical Rizzi et al. (2007) relationship, which has been applied by Jacobs et al. (2009) to determine distances to a large sample of galaxies in the Local Volume, and the recent Jang \& Lee (2017b) empirical calibration. The slope of the Rizzi et al. (2007) relationship was estimated by determining the apparent TRGB magnitude vs colour relation in a sample of galaxies, and averaging the slopes. The zero point determination was based on horizontal branch distances to IC 1613, NGC 185, the Sculptor and Fornax

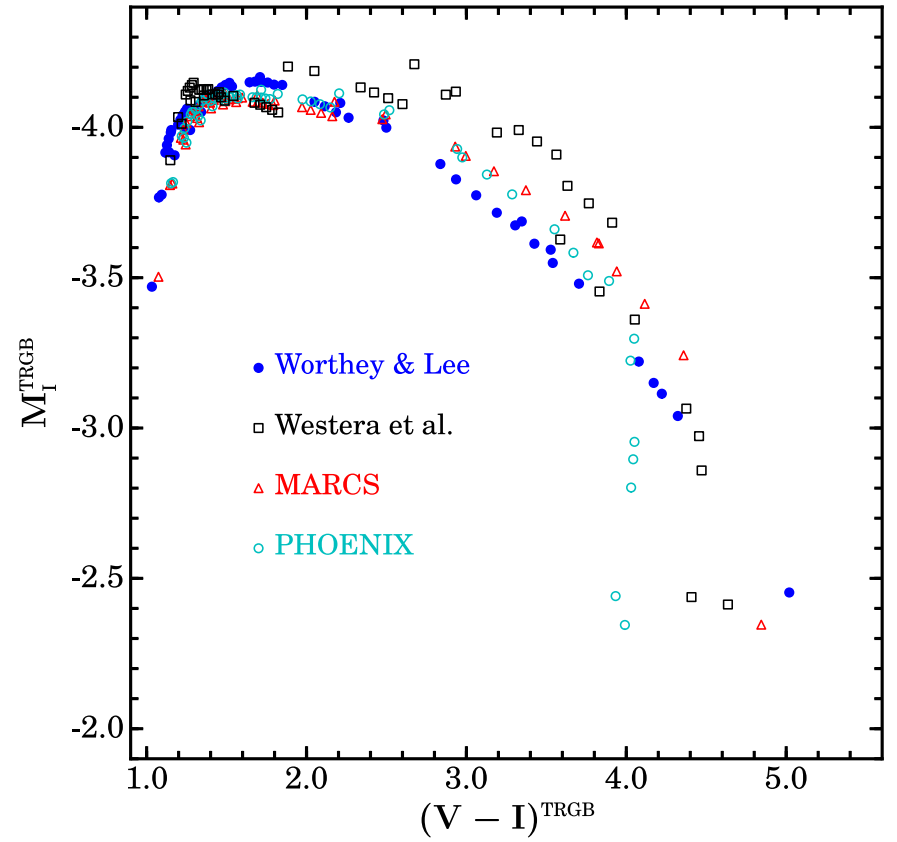

Fig. 8. Theoretical $M_{I}^{\mathrm{TRGB}}-(V-I)^{\mathrm{TRGB}}$ relation covering the full age and $[\mathrm{M} / \mathrm{H}]$ range of our reference calculations employing the four labelled sets of BCs.

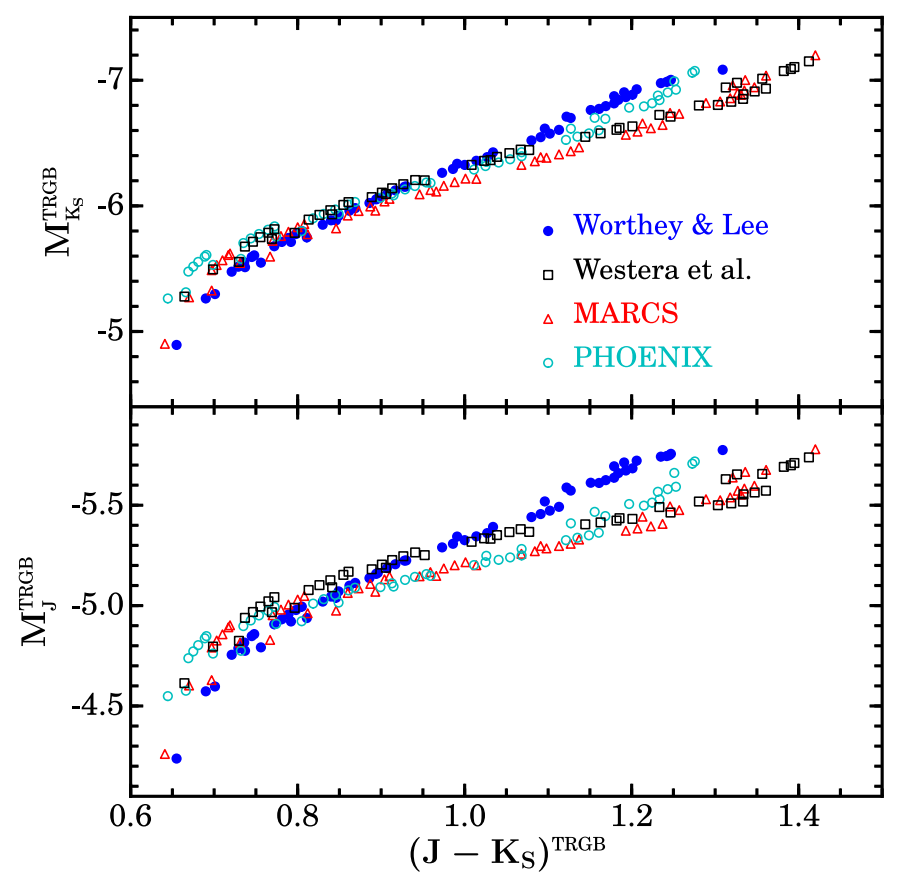

Fig. 9. As Fig. 8, but for the $M_{K_{\mathrm{s}}}^{\mathrm{TRGB}}-\left(J-K_{\mathrm{s}}\right)^{\mathrm{TRGB}}$ (upper panel) and $M_{J}^{\mathrm{TRGB}}-\left(J-K_{\mathrm{s}}\right)^{\mathrm{TRGB}}$ relations (lower panel) in the 2MASS photometric system.

dwarf spheroidal galaxies, and M33 based on the Carretta et al. (2000) HB absolute magnitude vs $[\mathrm{M} / \mathrm{H}]$ relationship. This relation was obtained by fitting main sequence distances to a sample of Galactic globular clusters using subdwarfs with Hipparcos parallaxes. The crucial difficulty here is to assign a $[\mathrm{M} / \mathrm{H}]$ to the $\mathrm{HB}$ stars in the galaxy. The authors employed a $(V-I)^{\mathrm{TRGB}}$ $[\mathrm{M} / \mathrm{H}]$ relationship based on Galactic globulars (see Rizzi et al. 2007 , for details) and assigned to the observed HB the $[\mathrm{M} / \mathrm{H}]$ derived from the measured $(V-I)^{\mathrm{TRGB}}$ colour. 


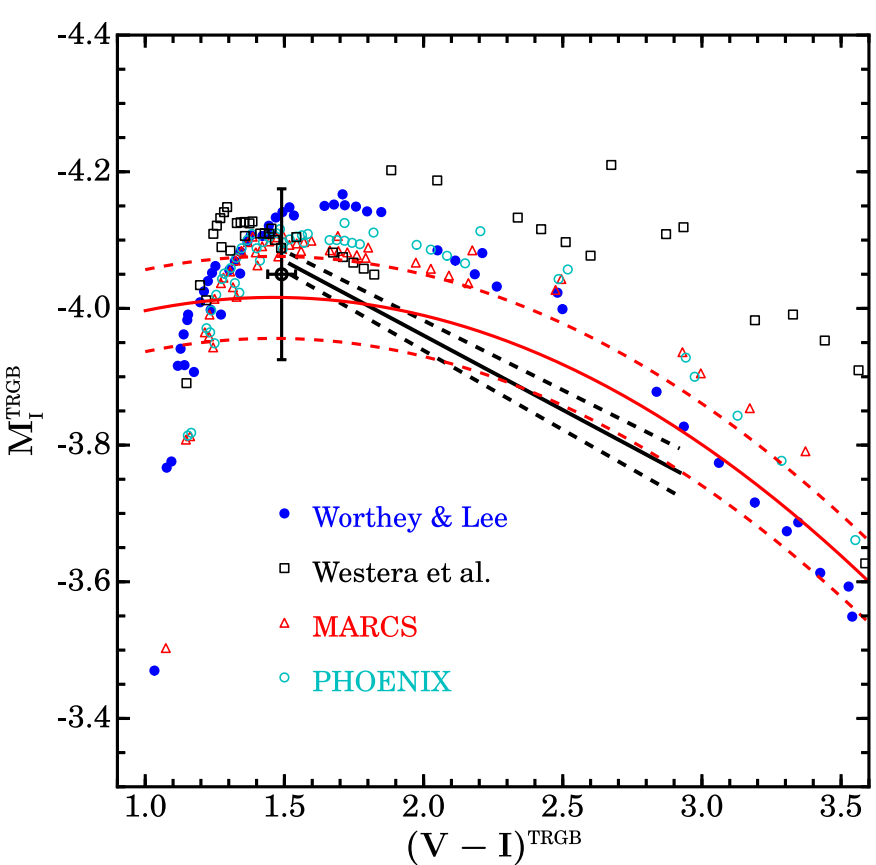

Fig. 10. As Fig. 8, but including the empirical value obtained for the Galactic globular cluster $\omega$ Cen (open circle with error bars), and the empirical relationships by Rizzi et al. (2007) and Jang \& Lee (2017b) based on samples of local galaxies (black and red lines, respectively; see text for details). The solid lines denote mean values of the relationships, while the dashed lines denote, respectively, the brighter and lower limits according to the $1 \sigma$ uncertainty on the slope and zero point of Rizzi et al. (2007) results, and the $1 \sigma$ uncertainty on the zero point of Jang \& Lee (2017b) calibration.

The quadratic form of the Jang \& Lee (2017b) calibration is also based on the apparent TRGB magnitude vs colour relations in a galaxy sample (different from Rizzi et al. 2007), with the zero point set by averaging the zero points obtained from the LMC (distance from eclipsing binaries) and NGC 4258 maser distance (the two zero points, however, are almost identical; see the paper for details). These two empirical calibrations are clearly different from the theoretical predictions, but also mutually different, especially in terms of the trend of the TRGB magnitude with colour.

Rizzi et al. (2007) TRGB magnitudes are generally fainter than the models, with a steeper slope compared to our results, irrespective of the adopted BCs. At $(V-I)^{\mathrm{TRGB}} 2.0-2.5$ differences in $M_{I}^{\mathrm{TRGB}}$ can reach up to $0.2-0.3 \mathrm{mag}$, depending on the chosen set of BCs. The calibration by Jang \& Lee (2017b) produces usually fainter TRGBs compared to the models (apart from the bluest colours), but the trend with colour, at least for $(V-I)^{\mathrm{TRGB}}>1.5$, is consistent with the theoretical calibration employing MARCS BCs.

For the infrared the situation is different. Figure 11 shows the $J K$ absolute magnitude-colour calibrations of Fig. 9 together with the empirical estimate for the Galactic globular cluster $\omega$ Cen, and the mean values for the LMC and SMC obtained from a number of fields within each galaxy. The last two estimates (from Górski et al. 2016) rely on the eclipsing binary distances to the Magellanic Clouds. The three constraints fall in the colour range where the differences due to the choice of BCs are minimized and, within the errors, are consistent with all four sets of BCs.

Finally, Fig. 12 shows the magnitude-colour calibrations in the WFC3-HST filters $F 110 W$ and $F 160 W$, similar to $J$ and

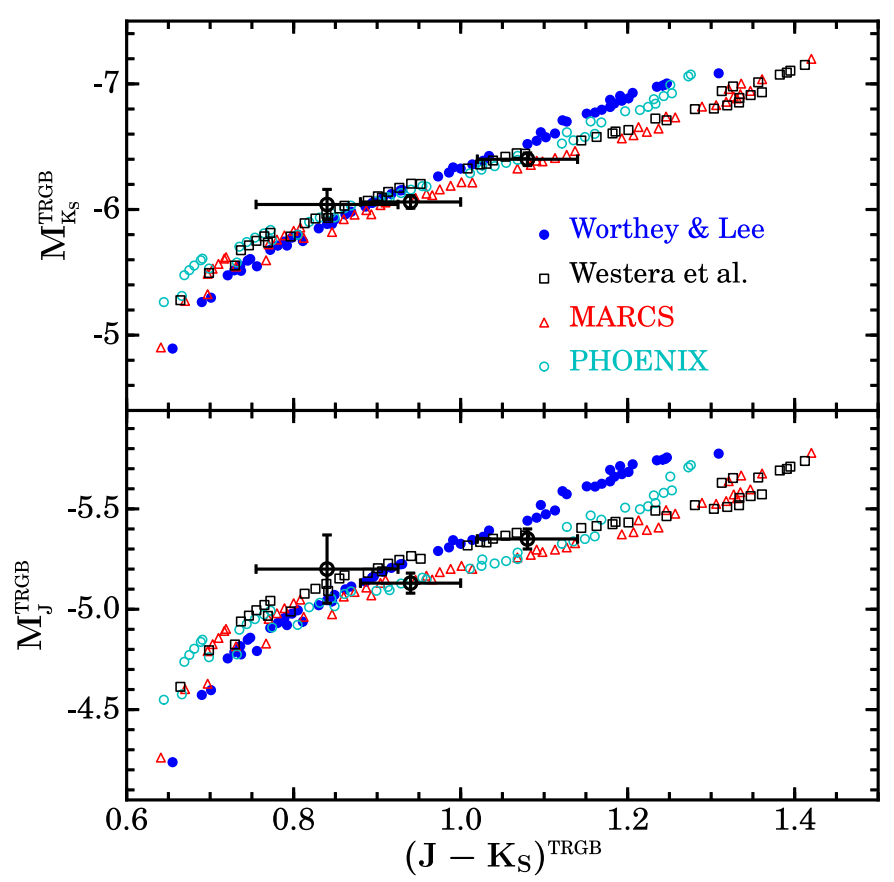

Fig. 11. As Fig. 9, but including the empirical values obtained for $\omega$ Cen, the SMC, and LMC.

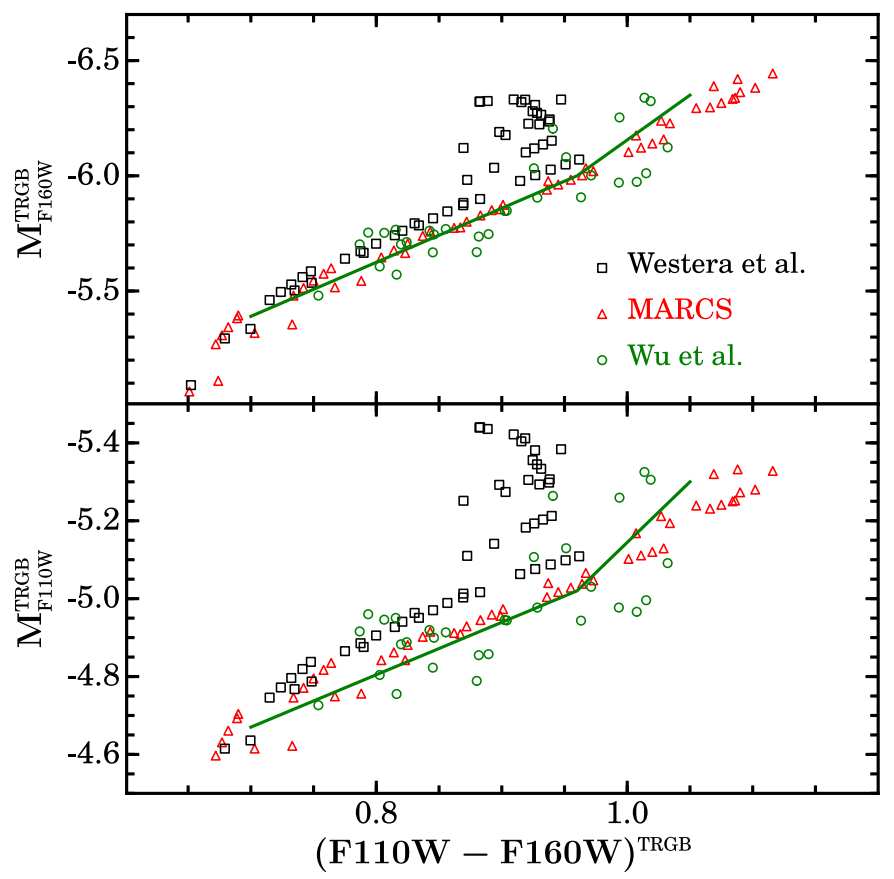

Fig. 12. As Fig. 9, but for the WFC3-HS T filters $F 110 \mathrm{~W}$ and $F 160 \mathrm{~W}$. We include the theoretical calibrations based on Westera et al. (2002) and MARCS BCs, and the empirical values (analytical formula plus the calibrating datapoints without error bars) by Wu et al. (2014).

$K$, compared to the empirical calibration by Wu et al. (2014) based on a sample of local galaxies, and fully consistent (in zero point) with the Rizzi et al. (2007) calibration in VI. We plot only theoretical results employing Westera et al. (2002) and MARCS BCs for we do not have PHOENIX and Worthey \& Lee (2011) $\mathrm{BCs}$ available in these filters. The theoretical results employing MARCS BCs are in good agreement with the empirical calibration. Some discrepancies appear at $(F 110 W-F 160 W)$ above $\sim 0.95$, where the empirical datapoints show a large dispersion. 
Table 1. Analytic fits to our reference calibrations of the RGB-tip magnitude-colour relations.

\begin{tabular}{|c|c|c|}
\hline$M_{I}^{\mathrm{TRGB}}$ & $\begin{array}{l}-4.090+0.086\left[(V-I)^{\mathrm{TRGB}}-1.4\right]+4.721\left[(V-I)^{\mathrm{TRGB}}-1.4\right]^{2} \\
-4.090+0.017\left[(V-I)^{\mathrm{TRGB}}-1.4\right]+0.036\left[(V-I)^{\mathrm{TRGB}}-1.4\right]^{2} \\
-4.037+0.087\left[(V-I)^{\mathrm{TRGB}}-2.4\right]+0.158\left[(V-I)^{\mathrm{TRGB}}-2.4\right]^{2}\end{array}$ & $\begin{array}{l}1.00<(V-I)^{\mathrm{TRGB}}<1.40 \\
1.40 \leq(V-I)^{\mathrm{TRGB}}<2.40 \\
2.40 \leq(V-I)^{\mathrm{TRGB}}<4.50\end{array}$ \\
\hline$M_{K_{\mathrm{S}}}^{\mathrm{TRGB}}$ & $\begin{array}{l}-5.722-2.386\left[(J-K)^{\mathrm{TRGB}}-0.76\right]+34.694\left[(J-K)^{\mathrm{TRGB}}-0.76\right]^{2} \\
-5.722-1.811\left[(J-K)^{\mathrm{TRGB}}-0.76\right]-0.517\left[(J-K)^{\mathrm{TRGB}}-0.76\right]^{2}\end{array}$ & $\begin{array}{l}0.60<(J-K)^{\mathrm{TRGB}}<0.76 \\
0.76 \leq(J-K)^{\mathrm{TRGB}}<1.50\end{array}$ \\
\hline$M_{J}^{\mathrm{TRGB}}$ & $\begin{array}{l}-4.962-1.386\left[(J-K)^{\mathrm{TRGB}}-0.76\right]+34.694\left[(J-K)^{\mathrm{TRGB}}-0.76\right]^{2} \\
-4.962-0.811\left[(J-K)^{\mathrm{TRGB}}-0.76\right]-0.517\left[(J-K)^{\mathrm{TRGB}}-0.76\right]^{2}\end{array}$ & $\begin{array}{l}0.60<(J-K)^{\mathrm{TRGB}}<0.76 \\
0.76 \leq(J-K)^{\mathrm{TRGB}}<1.50\end{array}$ \\
\hline$F 110 W^{\mathrm{TRGB}}=$ & $\begin{array}{l}-4.630-9.525\left[(F 110 W-F 160 W)^{\mathrm{TRGB}}-0.68\right] \\
-4.630-1.511\left[(F 110 W-F 160 W)^{\mathrm{TRGB}}-0.68\right]\end{array}$ & $\begin{array}{l}0.60<(F 110 W-F 160 W)^{\mathrm{TRGB}}<0.68 \\
0.68 \leq(F 110 W-F 160 W)^{\mathrm{TRGB}}<1.20\end{array}$ \\
\hline$F 160 W^{\mathrm{TRGB}}=$ & $\begin{array}{l}-5.310-10.525\left[(F 110 W-F 160 W)^{\mathrm{TRGB}}-0.68\right] \\
-5.310-2.511\left[(F 110 W-F 160 W)^{\mathrm{TRGB}}-0.68\right]\end{array}$ & $\begin{array}{l}0.60<(F 110 W-F 160 W)^{\mathrm{TRGB}}<0.68 \\
0.68 \leq(F 110 W-F 160 W)^{\mathrm{TRGB}}<1.20\end{array}$ \\
\hline
\end{tabular}

Notes. Calibrations are based on MARCS colours and BCs and are valid for $10^{-4} \leq Z \leq 0.04$ except for the $M_{I}^{\mathrm{TRGB}}$ vs. $(V-I)^{\mathrm{TRGB}}$ relation, valid up to $Z \approx 0.02$. Physical inputs in the models, including initial composition, are described in Sect. 3.2. Models do not include $\alpha$-enhancement (see text for discussion).

Wu et al. (2014) determined $1 \sigma$ dispersions around the mean relationship by 0.05 and $0.07 \mathrm{mag}$ in $F 110 \mathrm{~W}$ and $F 160 \mathrm{~W}$, respectively, when $(F 110 W-F 160 W)$ is below 0.95 . For larger colours the dispersions are equal to 0.12 and 0.09 mag in $F 110 \mathrm{~W}$ and $F 160 W$, respectively.

Considering together the comparisons of Figs. 11 and 12, the MARCS BCs seem to be well suited to be used for TRGB modelling in the infrared (up to metallicities around solar). The situation in the VI diagram is less clear; the Rizzi et al. (2007) results (which should be consistent with the IR empirical calibration discussed above) cannot be reproduced with any set of BCs, whilst the TRGB of $\omega$ Cen does not provide an additional strong constraint, due to the relatively large associated error bar. On the other hand, the alternative empirical calibration by Jang \& Lee (2017b) displays a trend with colour, for $(V-I)^{\mathrm{TRGB}}>1.5$, generally consistent with our models complemented by MARCS BCs, and a zero-point offset only very slightly larger than the $1 \sigma$ uncertainty (0.06 mag) associated with Jang \& Lee (2017b) TRGB magnitudes.

\subsection{Reference calibrations}

Based on discussions in previous sections, we present reference calibrations for the RGB tip in different colour-magnitude combinations. These are based on stellar models computed with the concordance input physics described in Sect. 3.2, short minimum time step (1 kyr; Sect. 3.3) and the MARCS BCs. Calibrations are presented in Table 1 as a set of analytic fits to facilitate their use. The dispersion between models and the fits are 0.02 for $M_{I}^{\mathrm{TRGB}}-(V-I)^{\mathrm{TRGB}}$ and 0.04 for the other relations. This is, in all cases, much smaller than the 0.12 uncertainty in the theoretical RGB-tip magnitudes discussed in Sect. 5.1.

For reference, Table 2 includes some properties of stellar models at the TRGB, including age, surface helium abundance $\left(Y_{\mathrm{S}}\right)$, luminosity, $T_{\mathrm{eff}}$, and $M_{V}^{\mathrm{TRGB}}$.

\section{Discussion}

The maximum brightness of low-mass stars along the RGB is a reference point in any CMD. It can be used for various purposes, ranging from absolute and relative distance determinations to non-standard cooling processes of degenerate cores, but also for age determinations of old populations, where its weak sensitivity to age can be used in determining distances and thus allow using other time-dependent CMD features to date it. To achieve a high accuracy in the results, one clearly needs an absolute calibration of the TRGB luminosity and its dependence -even if minor- on age and metallicity, both in terms of bolometric flux and magnitudes in photometric passbands. The present work is concerned with the theoretical prediction for the TRGB brightness, which depends both on the physics employed in the theoretical models, and on the numerical and technical details of stellar evolution codes. We have, for the first time, investigated a wide range of stellar masses (from 0.8 to $1.4 M_{\odot}$ ) and metallicities (from $1 \times 10^{-4}$ to $4 \times 10^{-2}$ ). This implies an age range from about 2 to 16 Gyr.

We started by comparing the results for models computed with nominally very similar standard physics using two standard evolutionary codes, GARSTEC (Weiss \& Schlattl 2008) and the BaSTI code (Pietrinferni et al. 2004). The results, presented in Sect. 3.1, are representative of the variation in theoretical predictions when using different model resources. They actually may represent a lower limit to the systematic uncertainties that should be taken into account when comparing models to observations. We showed that the differences can be appreciable for the lowest metallicities and higher masses, and larger than expected for the model dependencies that have been investigated to date and discussed in Sect. 2, which were almost exclusively done for older stars of masses below $1 M_{\odot}$.

We then adjusted the physics between the two codes, identifying the main sources for the differences (Sect. 3.2); these were the ${ }^{14} \mathrm{~N}(\mathrm{p}, \gamma){ }^{15} \mathrm{O} \mathrm{CNO}-\mathrm{cycle}$ bottleneck reaction and the electron screening of the $3 \alpha$ rate. With these adjustments, the differences shrank by up to a factor of 4 for the low-metallicity high-mass cases, but in fact increased for the highest metallicity.

In the next step numerical details were scrutinized (Sect. 3.3) and the temporal resolution on the upper RGB identified as the almost sole remaining issue. We determined the maximum time step allowed for converged resolution to be a few hundred years. This resulted in an overall excellent agreement between the two 
Table 2. Summary of TRGB properties for selected models.

\begin{tabular}{ccccccccc}
\hline \hline$Z$ & $Y$ & Mass & \multicolumn{1}{c}{ Age } & $M_{\mathrm{c}}^{\mathrm{He}}$ & $Y_{\mathrm{S}}$ & $\log L_{\mathrm{TRGB}}$ & $\log T_{\mathrm{eff}}^{\mathrm{TRGB}}$ & $M_{V}^{\mathrm{TRGB}}$ \\
\hline 0.0001 & 0.245 & 0.80 & 12.72 & 0.500 & 0.253 & 3.250 & 3.651 & -2.764 \\
$\prime \prime$ & $\prime \prime$ & 0.90 & 8.46 & 0.498 & 0.257 & 3.237 & 3.654 & -2.765 \\
$\prime \prime$ & $\prime \prime$ & 1.00 & 5.92 & 0.494 & 0.260 & 3.217 & 3.658 & -2.751 \\
\hline 0.0010 & 0.246 & 0.80 & 13.82 & 0.419 & 0.259 & 3.336 & 3.598 & -2.502 \\
$\prime \prime$ & $\prime \prime$ & 0.90 & 9.13 & 0.488 & 0.263 & 3.327 & 3.603 & -2.551 \\
$\prime \prime$ & $\prime \prime$ & 1.00 & 6.34 & 0.485 & 0.266 & 3.316 & 3.608 & -2.595 \\
\hline 0.0040 & 0.251 & 0.85 & 13.76 & 0.485 & 0.269 & 3.390 & 3.552 & -1.876 \\
$\prime \prime$ & $\prime \prime$ & 0.95 & 9.24 & 0.483 & 0.272 & 3.385 & 3.558 & -2.032 \\
$\prime \prime$ & $\prime \prime$ & 1.00 & 7.70 & 0.482 & 0.273 & 3.382 & 3.561 & -2.095 \\
\hline
\end{tabular}

model sets of the order of 0.1 dex in $\log \left(L_{\mathrm{TRGB}} / L_{\odot}\right)$ except, again, for the higher masses at the lowest metallicity (Fig. 3). We could not resolve this difference, but it may be related to the different evolution of very small convective cores on the main sequence, the only obvious difference we found for these models, and may be related to the detailed treatment of convective boundaries (see also Gabriel et al. 2014). Fortunately, such stars are not relevant, at least in the Milky Way, because of the combination of their young age and very low metallicity. Nevertheless, this issue requires further investigations beyond the goal of the present paper.

With the physics and numerics that resulted in an overall excellent agreement of the TRGB luminosities derived from both codes, we computed and present in Sect. 4 a set of models, which we recommend as a reference for further applications of the TRGB in astrophysics. We used canonical, but nevertheless upto-date physics, ignoring mass-loss, diffusion, extra-mixing on the RGB, and rotation. However, all these potentially interesting effects have a much larger theoretical uncertainty than, for example, the equation of state or electron conduction opacities, and therefore constitute a much greater degree of freedom for modellers and their output.

In the second part of the paper (Sect. 5) we transposed results for the TRGB $L$ and $T_{\text {Teff }}$ into magnitudes and colours, in particular red and infrared magnitudes, as they are known to be best suited for employing the TRGB as distance indicator. For our reference set of models we show the global relations between TRGB brightness and colour in $V I$ and $J K_{\mathrm{s}}$ diagrams (Figs. 5 and 6, respectively) based on one particular theoretical set of bolometric corrections (Worthey \& Lee 2011). Comparisons of results obtained with several $\mathrm{BC}$ sets show that the uncertainties arising from $\mathrm{BC}$ calculations (Sect. 5.2) in fact exceed those from the stellar models. In infrared colours, the use of MARCS BCs gives results that seem to agree most closely with an existing empirical relation, although the empirical database is not yet stringent enough for a final conclusion. In the VI diagram the existing empirical relations are not in mutual agreement, and it is very hard to assess which set of BCs, if any, is best suited for a theoretical calibration.

The comparison of our theoretical predictions with the recent work by Viaux et al. (2013b), to which we refered in the discussion of known dependencies (Sect. 2), shows some noteworthy differences. They find a theoretical $M_{I}=-3.99$ for stellar models appropriate for the globular cluster M 5. Using matching models from our reference set, we find $M_{I}=-4.14$ with the Worthey \& Lee (2011) transformations, the ones also used by Viaux et al. (2013b). This difference translates into $\Delta \log \left(L_{\mathrm{TRGB}} / L_{\odot}\right) \approx 0.06 \mathrm{dex}$, with our models being more luminous. This is confirmed by comparing our results with their
Fig. 6. We trace part of the difference, 0.035 dex, to the treatment of electron screening, for which Viaux et al. (2013b) use Salpeter's formulation of weak screening, whereas our reference set of models includes the intermediate screening regime, appropriate for RGB stars. We emphasize again that screening in the weak regime is in fact stronger than in the intermediate regime (Sect. 2.2), leading to a dimmer RGB tip. Much of the physics used in our models and those of Viaux et al. (2013b) is the same so it is not easy to identify the cause for the remaining $0.025 \mathrm{dex}$ difference. It is possible, however, that it might be due to numerical aspects of the calculations that, as we have found in this work, can have a strong impact on the theoretical $L_{\text {TRGB }}$ predictions. Our results strongly alleviate the tension between predictions of standard stellar models for the RGB tip brightness and observations of M 5 found by Viaux et al. (2013b). We predict $M_{I}=-4.14 \pm 0.12$ or $-4.07 \pm 0.12$ for the RGB tip depending on whether the Worthey \& Lee (2011) or MARCS BCs are used, well within the $1 \sigma$ observational band $M_{I}=-4.17 \pm 0.13$ determined by Viaux et al. (2013b) for M 5 that is dominated by a 0.11 uncertainty in the distance modulus. This would basically remove any need for additional cooling, and therefore sets very low limits on a possible magnetic dipole moment of neutrinos or the mass of axions, as investigated by Viaux et al. (2013b) and Viaux et al. (2013a).

The cosmological significance of a careful calibration of the TRGB brightness is exemplified by the following. Recently, Jang \& Lee (2017a) re-calibrated the Hubble constant $H_{0}$ using the TRGB brightness from their own empirical calibration for deriving the absolute brightness of type Ia supernovae. They arrived at a value of $71.17 \pm 2.03$ (rand.) \pm 1.94 (syst.) $\mathrm{km} \mathrm{s}^{-1} \mathrm{Mpc}^{-1}$, slightly lower than previous Cepheid-based determinations, but still in conflict with the value obtained from the most recent CMB analysis (Planck Collaboration Int. XLVI 2016, $H_{0}=$ $\left.66.93 \mathrm{~km} \mathrm{~s}^{-1} \mathrm{Mpc}^{-1}\right)$. Since their calibration is, depending on colour transformation, fainter than our theoretical prediction by 0.05-0.10 mag (see Fig. 10), a change to our calibration would increase the distance scale and decrease $H_{0}$ by $1-2 \%$ to a value between $\approx 68 \ldots 70 \mathrm{~km} \mathrm{~s}^{-1} \mathrm{Mpc}^{-1}$.

We conclude that it is possible, by a careful investigation into the details of numerical stellar model computations, to produce a reference set of accurate predictions for the TRGB luminosity. The major uncertainty in applying this to CMDs, either of simple or composite stellar populations, appears to lie in the BC scale. Much work is needed here to achieve the same confidence as in the underlying stellar models.

Acknowledgements. The authors thank the anonymous referee for the effort in preparing an extensive, knowledgeable, and constructive report that has 
contributed to improving this work. We also want to thank Nicolás Viaux for providing original data and for helpful discussions. A.S. is partially supported by ESP2015-66134-R (MINECO). S.C. acknowledges partial financial support from PRIN-INAF2014 (PI: S. Cassisi) and from grant AYA2016-77237-C3-1-P from the Spanish Ministry of Economy and Competitiveness (MINECO). A.W. and M.S. are grateful for the hospitality of the Lorentz Centre in Leiden, where the initial discussions about this subject took place during one of their workshops. This research was supported by the Munich Institute for Astro- and Particle Physics (MIAPP) of the DFG cluster of excellence "Origin and Structure of the Universe".

\section{References}

Adelberger, E. G., García, A., Robertson, R. G. H., et al. 2011, Rev. Mod. Phys., 83,195

Angulo, C., Arnould, M., Rayet, M., et al. 1999, Nucl. Phys. A, 656, 3

Arceo-Díaz, S., Schröder, K.-P., Zuber, K., \& Jack, D. 2015, Rev. Mex. Astron. Astrofis., 51, 151

Baade, W. 1944, ApJ, 100, 137

Barker, M. K., Sarajedini, A., \& Harris, J. 2004, ApJ, 606, 869

Bellazzini, M., Ferraro, F. R., \& Pancino, E. 2001, ApJ, 556, 635

Bellazzini, M., Ferraro, F. R., Sollima, A., Pancino, E., \& Origlia, L. 2004, A\&A, 424, 199

Carpenter, J. M. 2001, AJ, 121, 2851

Carretta, E., Gratton, R. G., Clementini, G., \& Fusi Pecci, F. 2000, ApJ, 533, 215

Casagrande, L., \& VandenBerg, D. A. 2014, MNRAS, 444, 392

Cassisi, S. 2010, in Stellar Populations - Planning for the Next Decade, eds.

G. R. Bruzual, \& S. Charlot, IAU Symp., 262, 13

Cassisi, S., \& Salaris, M. 2013, Old Stellar Populations: How to Study the Fossil Record of Galaxy Formation (Wiley)

Cassisi, S., Castellani, V., degl'Innocenti, S., \& Weiss, A. 1998, A\&AS, 129, 267

Cassisi, S., Salaris, M., \& Irwin, A. W. 2003, ApJ, 588, 862

Cassisi, S., Salaris, M., Castelli, F., \& Pietrinferni, A. 2004, ApJ, 616, 498

Cassisi, S., Potekhin, A. Y., Pietrinferni, A., Catelan, M., \& Salaris, M. 2007, ApJ, 661, 1094

Castellani, V., \& degl'Innocenti, S. 1993, ApJ, 402, 574

Catelan, M. 2013, in European Physical Journal Web of Conferences, 43, 01001

Catelan, M., de Freitas Pacheco, J. A., \& Horvath, J. E. 1996, ApJ, 461, 231

Cioni, M.-R. L., van der Marel, R. P., Loup, C., \& Habing, H. J. 2000, A\&A, 359,601

Conn, A. R., Lewis, G. F., Ibata, R. A., et al. 2011, ApJ, 740, 69

Cyburt, R. H., Fields, B. D., Olive, K. A., \& Yeh, T.-H. 2016, Rev. Mod. Phys. 88,015004

Da Costa, G. S., \& Armandroff, T. E. 1990, AJ, 100, 162

Delahaye, F., \& Pinsonneault, M. H. 2006, ApJ, 649, 529

Dewitt, H. E., Graboske, H. C., \& Cooper, M. S. 1973, ApJ, 181, 439

Dotter, A., Chaboyer, B., Jevremović, D., et al. 2008, ApJS, 178, 89

Ferguson, J. W., Alexander, D. R., Allard, F., et al. 2005, ApJ, 623, 585

Formicola, A., Imbriani, G., Costantini, H., et al. 2004, Phys. Lett. B, 591, 61

Fynbo, H. O. U., Diget, C. A., Bergmann, U. C., et al. 2005, Nature, 433, 136

Gabriel, M., Noels, A., Montalbán, J., \& Miglio, A. 2014, A\&A, 569, A63

Górski, M., Pietrzyński, G., Gieren, W., et al. 2016, AJ, 151, 167

Graboske, H. C., Dewitt, H. E., Grossman, A. S., \& Cooper, M. S. 1973, ApJ 181,457
Grevesse, N., \& Noels, A. 1993, in Origin and Evolution of the Elements, Symp. in honour of $\mathrm{H}$. Reeves 60 th birthday, eds. N. Prantzos, E. Vangioni-Flam, \& M. Casse, 15

Gustafsson, B., Edvardsson, B., Eriksson, K., et al. 2008, A\&A, 486, 951

Haft, M., Raffelt, G., \& Weiss, A. 1994, ApJ, 425, 222

Iglesias, C. A., \& Rogers, F. J. 1996, ApJ, 464, 943

Imbriani, G., Costantini, H., Formicola, A., et al. 2004, A\&A, 420, 625

Itoh, N., Hayashi, H., Nishikawa, A., \& Kohyama, Y. 1996a, ApJS, 102, 411

Itoh, N., Nishikawa, A., \& Kohyama, Y. 1996b, ApJ, 470, 1015

Jacobs, B. A., Rizzi, L., Tully, R. B., et al. 2009, AJ, 138, 332

Jang, I. S., \& Lee, M. G. 2017a, ApJ, 836, 74

Jang, I. S., \& Lee, M. G. 2017b, ApJ, 835, 28

Kippenhahn, R., Weigert, A., \& Weiss, A. 2012, Stellar Structure and Evolution (Berlin, Heidelberg: Springer-Verlag)

Lee, M. G., Freedman, W. L., \& Madore, B. F. 1993, ApJ, 417, 553

Madore, B. F., \& Freedman, W. L. 1995, AJ, 109, 1645

Maíz-Apellániz, J., Cieza, L., \& MacKenty, J. W. 2002, AJ, 123, 1307

Makarov, D., Makarova, L., Rizzi, L., et al. 2006, AJ, 132, 2729

Michaud, G., Richer, J., \& Richard, O. 2010, A\&A, 510, A104

Munakata, H., Kohyama, Y., \& Itoh, N. 1985, ApJ, 296, 197

Pietrinferni, A., Cassisi, S., Salaris, M., \& Castelli, F. 2004, ApJ, 612, 168

Pietrinferni, A., Cassisi, S., \& Salaris, M. 2010, A\&A, 522, A76

Planck Collaboration Int. XLVI. 2016, A\&A, 596, A107

Potekhin, A. Y. 1999, A\&A, 351, 787

Raffelt, G. G. 1990, ApJ, 365, 559

Raffelt, G., \& Weiss, A. 1992, A\&A, 264, 536

Raffelt, G., \& Weiss, A. 1995, Phys. Rev. D, 51, 1495

Refsdal, S., \& Weigert, A. 1970, A\&A, 6, 426

Richard, O., Michaud, G., Richer, J., et al. 2002, ApJ, 568, 979

Rizzi, L., Tully, R. B., Makarov, D., et al. 2007, ApJ, 661, 815

Sakai, S., Madore, B. F., \& Freedman, W. L. 1996, ApJ, 461, 713

Salaris, M., \& Cassisi, S. 1997, MNRAS, 289, 406

Salaris, M., \& Cassisi, S. 1998, MNRAS, 298, 166

Salaris, M., \& Cassisi, S. 2006, Evolution of Stars and Stellar Populations (Wiley)

Salaris, M., \& Girardi, L. 2005, MNRAS, 357, 669

Salaris, M., Chieffi, A., \& Straniero, O. 1993, ApJ, 414, 580

Salaris, M., Cassisi, S., \& Weiss, A. 2002, PASP, 114, 375

Salpeter, E. E. 1954, Aust. J. Phys., 7, 373

Sandage, A. 1971, ApJ, 166, 13

Thoul, A. A., Bahcall, J. N., \& Loeb, A. 1994, ApJ, 421, 828

Valle, G., Dell'Omodarme, M., Prada Moroni, P. G., \& Degl'Innocenti, S. 2013, A\&A, 549, A50

VandenBerg, D. A., Bergbusch, P. A., Dotter, A., et al. 2012, ApJ, 755, 15

Viaux, N., Catelan, M., Stetson, P. B., et al. 2013a, Phys. Rev. Lett., 111, 231301

Viaux, N., Catelan, M., Stetson, P. B., et al. 2013b, A\&A, 558, A12

Villante, F. L., Serenelli, A. M., Delahaye, F., \& Pinsonneault, M. H. 2014, ApJ, 787,13

Vinyoles, N., Serenelli, A. M., Villante, F. L., et al. 2017, ApJ, 835, 202

Weiss, A., \& Schlattl, H. 2008, Ap\&SS, 316, 99

Weiss, A., Serenelli, A., Kitsikis, A., Schlattl, H., \& Christensen-Dalsgaard, J. 2005, A\&A, 441, 1129

Westera, P., Lejeune, T., Buser, R., Cuisinier, F., \& Bruzual, G. 2002, A\&A, 381, 524

Worthey, G., \& Lee, H.-c. 2011, ApJS, 193, 1

Wu, P.-F., Tully, R. B., Rizzi, L., et al. 2014, AJ, 148, 7 